University of Nebraska - Lincoln

DigitalCommons@University of Nebraska - Lincoln

Faculty Publications from the Harold W. Manter Laboratory of Parasitology

2011

Nematode Parasites of Costa Rican Snakes (Serpentes) with Description of a New Species of Abbreviata (Physalopteridae)

Charles R. Bursey

Pennsylvania State University - Shenango, cxb13@psu.edu

Daniel R. Brooks

University of Toronto, dnlbrooks@gmail.com

Follow this and additional works at: https://digitalcommons.unl.edu/parasitologyfacpubs

Part of the Parasitology Commons

Bursey, Charles R. and Brooks, Daniel R., "Nematode Parasites of Costa Rican Snakes (Serpentes) with Description of a New Species of Abbreviata (Physalopteridae)" (2011). Faculty Publications from the Harold W. Manter Laboratory of Parasitology. 695.

https://digitalcommons.unl.edu/parasitologyfacpubs/695

This Article is brought to you for free and open access by the Parasitology, Harold W. Manter Laboratory of at DigitalCommons@University of Nebraska - Lincoln. It has been accepted for inclusion in Faculty Publications from the Harold W. Manter Laboratory of Parasitology by an authorized administrator of DigitalCommons@University of Nebraska - Lincoln. 


\title{
Nematode Parasites of Costa Rican Snakes (Serpentes) with Description of a New Species of Abbreviata (Physalopteridae)
}

\author{
Charles R. Bursey ${ }^{1,3}$ and Daniel R. Brooks ${ }^{2}$ \\ ${ }^{1}$ Department of Biology, Pennsylvania State University, Shenango Campus, Sharon, Pennsylvania 16146, U.S.A. \\ (e-mail: cxb13@psu.edu) and \\ ${ }^{2}$ Department of Ecology and Evolutionary Biology, University of Toronto, Ontario, Canada M5S 3G5
}

\begin{abstract}
Seventy-eight snakes collected in the Area de Conservación Guanacaste, Costa Rica were necropsied: Boidae (1 species), boa constrictor, Boa constrictor; Colubridae (21 species), Ecuador sipo, Chironius grandisquamis; road guarder, Conophis lineatus; South American forest racer, Dendrophidion percarinatus; speckled racer, Drymobius margaritiferus; mimic false coral snake, Erythrolamprus mimus; blunthead tree snake, Imantodes cenchoa; western tree snake, Imantodes inornatus; banded cat-eyed snake, Leptodeira annulata; striped lowland snake, Leptodrymus pulcherrimus; Cope's parrot snake, Leptophis depressirostris; yellow-bellied racer, Liophis epinephelus; neotropical whip snake, Masticophis mentovarius; brown vinesnake, Oxybelis aeneus; Cope's vine snake, Oxybelis brevirostris; green vine snake, Oxybelis fulgidus; Cope's false coral snake, Pliocercus euryzonus; cloudy snail-eating snake, Sibon nebulata; chicken snake, Spilotes pullatus; western lyre snake, Trimorphodon biscutatus; orange-bellied littersnake, Urotheca guentheri; false fer-de-lance, Xenodon rabdocephalus; Viperidae (6 species), cantil, Agkistrodon bilineatus; jumping pit viper, Atropoides nummifer; terciopelo, Bothrops asper; cascabel, Crotalus durissus; hognosed pit viper, Porthidium nasutum; and slender hognose viper, Porthidium ophryomegas. Gravid nematodes representing 16 known species, Aplectana incerta, Aplectana itzocanensis, Cosmocercoides variabilis, Cruzia rudolphi, Hastospiculum onchocercum, Hexametra boddaertii, Kalicephalus costatus, Kalicephalus inermis, Kalicephalus sublatus, Macdonaldius oscheri, Ophidascaris arndti, Ophidascaris sicki, Physaloptera retusa, Skrjabinelazia intermedia, Terranova caballeroi, Travassosascaris araujoi, 1 new species, Abbreviata costaricae, and larvae of a species of Porrocaecum were found. Twenty-six new host records and 4 new locality records are reported. The nematode species found in this study are generalist parasites, i.e., infect more than one host species, and a comprehensive review of the hosts of these species is presented.
\end{abstract}

KEY WORDS: snakes, Costa Rica, Nematoda.

Little information about nematode parasites of Costa Rican snakes is available. The earliest published work on nematodes of Costa Rican snakes was that of Viquez (1935), who reported Physaloptera obtussissima in fer-de-lance, Bothrops atrox and unidentified species of Aplectana, Ophidascaris, and Thelazia in boa constrictor, Boa constrictor. The next reports were those of Brenes and Bravo-Hollis (1960), Kalicephalus macrovulvus (currently Kalicephalus inermis macrovulvus) in Bothrops atrox, and Schad (1962), Kalicephalus costatus and Kalicephalus subulatus in Boa constrictor. More recently, Goldberg and Bursey (2004a, 2005a, 2006a, b, 2009a, b) have reported adults of Hastospiculum onchocercum, Hexametra boddaertii, Terranova caballeroi, and larvae of Contracaecum sp., Ophidascaris sp., and Porrocaecum sp. in Costa Rican snakes. Our work on Costa Rican snakes has produced additional hosts as well as a new nematode species, Abbreviata costaricae, which we present herein.

\footnotetext{
${ }^{3}$ Corresponding author.
}

\section{MATERIALS AND METHODS}

Seventy-eight snakes representing 3 families collected in the Area de Conservación Guanacaste, Costa Rica were necropsied: Boidae: boa constrictor, Boa constrictor Linnaeus, 1758 (4); Colubridae: Ecuador sipo, Chironius grandisquamis Peters, 1869 (1); road guarder, Conophis lineatus (Duméril, Bibron and Duméril, 1854) (1); South American forest racer, Dendrophidion percarinatus Cope, 1893 (1); speckled racer, Drymobius margaritiferus Schlegel, 1837 (5); mimic false coral snake, Erythrolamprus mimus Cope, 1868 (2); blunthead tree snake, Imantodes cenchoa Linnaeus, 1758 (5); western tree snake, Imantodes inornatus Boulenger, 1896 (1); banded cat-eyed snake, Leptodeira annulata Linnaeus, 1758 (4); striped lowland snake, Leptodrymus pulcherrimus Cope, 1874 (1); Cope's parrot snake, Leptophis depressirostris Cope, 1861 (4); yellow-bellied racer, Liophis epinephelus (1); neotropical whip snake, Masticophis mentovarius Duméril, Bibron and Duméril, 1854 (1); brown vinesnake, Oxybelis aeneus Wagler, 1824 (5); Cope's vine snake, Oxybelis brevirostris Cope, 1861 (2); green vine snake, Oxybelis fulgidus Daudin, 1803 (3); Cope's false coral snake, Pliocercus euryzonus Cope, 1862 (1); cloudy snail-eating snake, Sibon nebulata Linnaeus, 1758 (2); chicken snake, Spilotes pullatus Linnaeus, 1758 (2); western lyre snake, Trimorphodon biscutatus Duméril, Bibron and Duméril, 1854 (7); orangebellied littersnake, Urotheca guentheri Dunn, 1938 (1); false fer-de-lance, Xenodon rabdocephalus Wied, 1924 (1); Viperidae: Cantil, Agkistrodon bilineatus Günther, 1863 
(2); jumping pit viper, Atropoides nummifer Rüppell, 1845 (1); terciopelo, Bothrops asper Garman, 1883 (2); cascabel, Crotalus durissus Linnaeus, 1758 (11); hognosed pit viper, Porthidium nasutum Bocourt, 1868 (1); and slender hognose viper, Porthidium ophryomegas Bocourt, 1868 (6).

Snakes had been collected by hand. During necropsy, parasites were separated by taxonomic class and sent to various laboratories for further study and identification. Only nematodes were received at this laboratory. Nematodes had been fixed in glacial acetic acid and preserved and stored in $70 \%$ ethanol. For examination and identification, they were cleared in lactophenol and examined by light microscopy. Measurements are given in micrometers unless otherwise stated, with mean $\pm 1 \mathrm{SD}$ and range in parenthesis. Drawings were made with the aid of a microprojector (Model 358B, Bausch and Lomb, Rochester, New York). Selected nematodes were deposited in the United States National Parasite Collection (USNPC), Beltsville, Maryland. Snake taxonomy follows Savage (2002).

\section{RESULTS}

Gravid nematodes representing 16 known species, Aplectana incerta Caballero, 1949, Aplectana itzocanensis Bravo Hollis, 1943, Cosmocercoides variabilis (Harwood, 1930), Cruzia rudolphi Ruiz, 1947, Hastospiculum onchocercum Chitwood, 1932, Hexametra boddaertii (Baird, 1860), Kalicephalus costatus (Rudolphi, 1819), Kalicephalus inermis (Molin, 1861), Kalicephalus sublatus Molin, 1861, Macdonaldius oscheri Chabaud and Frank, 1961, Ophidascaris arndti Sprehn, 1929, Ophidascaris sicki Freitas, 1951, Physaloptera retusa Rudolphi, 1819, Skrjabinelazia intermedia (Freitas, 1940), Terranova caballeroi Barus and Coy Otero, 1966, Travassosascaris araujoi Sprent, 1978, 1 new species, Abbreviata costaricae, and larvae of a species of Porrocaecum were found.

Thirty-three of 78 snakes ( $42 \%$ ) were infected with a total of 309 nematodes representing 18 species. Nematodes were not found in Bothrops asper, Leptodeira annulata, or Pliocercus euryzonus. No host snake harbored more than 2 nematode species: 22 hosts harbored 1 species, 11 hosts harbored 2 species; there were $1.3 \pm 0.5 \mathrm{SD}$ (range 1-2) nematode species per host individual and $9.4 \pm 12.9$ (range 1-53) nematodes per host. No snake species harbored more than 4 nematode species; there were $1.6 \pm 0.8$ (range 1-4) nematode species per snake species. Twenty-six new host records and 4 new locality records are reported.

\section{Abbreviata costaricae n. sp. Figures 1-8}

\section{Description}

General: Spirurida. Physalopteridae (Railliet, 1893) Leiper, 1908; Abbreviata Travassos, 1920. Medium- sized nematodes, body cylindrical, width similar throughout. Head with two lateral pseudolabia each bearing two small submedian papillae and one amphid. Each pseudolabium armed with a large externolateral tooth and 2 doubled submedian teeth, internolateral tooth and denticles absent. Head with distinct cervical cuticular inflation. Short buccal cavity; esophagus divided into short anterior muscular portion and long posterior glandular portion. Nerve ring slightly anterior to junction of muscular and glandular portions of esophagus. Deirids at level of nerve ring. Inconspicuous excretory pore slightly posterior to junction of muscular and glandular portions of esophagus.

Male (holotype and 2 paratypes): Length $18.17 \pm$ $2.02 \mathrm{~mm}(16.0-20.0 \mathrm{~mm})$; width at level of esophago-intestinal junction $400 \pm 53$ (357-459). Vestibule $49 \pm 6$ (43-55). Esophagus 2,133 \pm 98 $(2,048-2,240)$ in total length, divided into short muscular portion $236 \pm 21$ (214-256) in length, glandular portion $1,897 \pm 78(1,834-1,984)$ in length. Nerve ring $248 \pm 27$ (220-275) from anterior end. Spicules unequal. Right spicule well sclerotized, short, $183 \pm 7$ (179-191) long; left spicule, less sclerotized, sinuous, $1,505 \pm 159(1,326-1,632)$ in length, approximately 8 times length of right, terminating in fine point. Tail conical with rounded terminus. Caudal bursa well developed; 4 pairs of pendunculate papillae supporting bursal alae, 2 pair precloacal, 2 pairs postcloacal; 11 sessile and 2 mammiliform papillae, 3 sessile papillae precloacal, 1 on midline of anterior cloacal lip, 2 more anterior, positioned at right and left edges of cloaca; 4 sessile papillae in 2 pairs immediately posterior to cloaca, 4 papillae, 2 sessile, 2 mammiliform halfway between cloaca and end of tail; 2 sessile near end of tail. Ventral surface of posterior end with many small ovate verrucae beginning anterior to anteriormost pair of penduculate papillae and extending posterior to third pair of post anal sessile papillae.

Female (allotype and 4 paratypes): Length $25.10 \pm$ $2.66 \mathrm{~mm}(22.0-29.0 \mathrm{~mm})$; width at level of esophagointestinal junction $456 \pm 43(408-510)$. Vestibule 57 \pm 7 (49-66). Esophagus 2,406 $\pm 167(2,240-2,624)$ total length; divided into short muscular portion $277 \pm$ 20 (250-305) in length, glandular portion 2,133 \pm 147 $(1,980-2,319)$ in length. Nerve ring $255 \pm 14$ (240$275)$ and vulva 5,184 $\pm 239(4,864-5,376)$ from anterior end. Vulva on slight prominence, thin walled vagina directed posteriorly, opening into egg reservoir, then dividing dichotomously to give rise to four uteri. Eggs thick-walled, ovoid, embryonated, $42 \pm 4$ (37- 

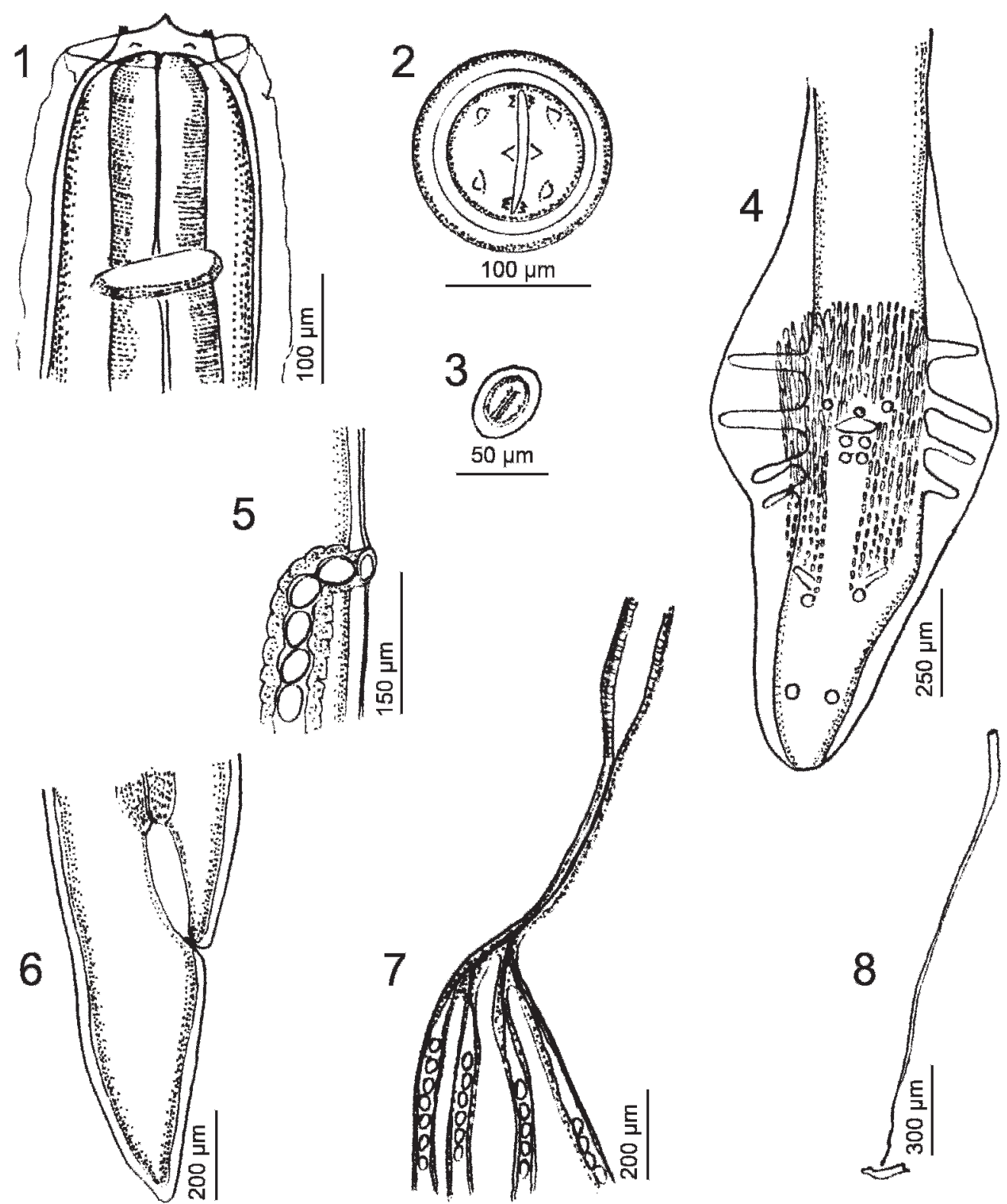

Figures 1-8. Abbreviata costaricae n. sp. 1. Female, anterior end, lateral view. 2. Female, en face view. 3. Egg. 4. Male, posterior end, ventral view. 5. Female, vulvar region. 6. Female, posterior end. 7. Female, uterine branches. 8. Male, spicules.

49) $\times 32 \pm 3(27-37)$. Tail $538 \pm 29(510-587)$ in length, rounded terminus.

\section{Taxonomic Summary}

Type host: Agkistrodon bilineatus Günther, 1863, cantil, symbiotype UT 03-P-0399, male, collected 25 May 2003.
Type locality: Guanacaste Conservation Area, Costa Rica.

Site of infection: Stomach.

Type specimens: Holotype male USNPC 103603; allotype female USNPC 103604; paratypes USNPC 103605 . 
Etymology: The new species is named for its collection locality.

\section{Remarks}

Of the known species of Abbreviata, only 3 have been reported from the Americas, namely Abbreviata bahamensis Moravec, Coy Otero and Barus, 1990 in a lizard, Leiocephalus sp. collected on Castle Island, Bahama; Abbreviata baracoa Barus and Coy Otero, 1966 in a snake, Alsophis cantherigerus collected in Cuba; Abbreviata terrapenis (Hill, 1941) Morgan, 1945 in a tortoise, Terapene ornate collected in Oklahoma, U.S.A. (Hill, 1941; Barus and Coy Otero, 1966; Moravec et al., 1990). Abbreviata costaricae $\mathrm{n}$. sp. is easily differentiated from A. bahamensis and $A$. baracoa species by uterine anatomy; both of these species have 2 uterine branches. Females of both Abbreviata costaricae n. sp. and A. terrapenis possess a uterus with 4 branches; however, the verrucae on the ventral surface of the bursa extend beyond the fifth postcloacal papillae in A. terrapenis but end near the third postcloacal papillae in $A$. costaricae.

\section{ASCARIDIDA}

\section{Cosmocercoidea (Railliet, 1916, subfam) Travassos, 1925 Cosmocercinae Railliet, 1916 Aplectana incerta Caballero, 1949}

(Syn. Oxysomatium incertum [Caballero, 1949] Skrjabin, Schikhobalova and Mozgovoi, 1951.)

Hosts and locality: Chironius grandisquamis (1): male, UT 03-P-0241, collected 14 March 2003, Rio Mena, Guanacaste Conservation Area; Conophis lineatus (1): UT 01-P-0011, collected 4 June 2001, Area Administrative, Guanacaste Conservation Area; Porthidium nasutum (1): male, UT 03-P-0400, collected 25 May 2003, San Gerardo, Guanacaste Conservation Area; Porthidium ophryomegas (1): female, UT 04-P-0438, collected 14 June 2004, Centeno, Guanacaste Conservation Area.

Prevalence and intensity: 1 of $1 C$. grandiaquamis (100\%, 1$)$; 1 of 1 C. lineatus $(100 \%, 16) ; 1$ of $1 P$. nasutum $(100 \%, 34) ; 1$ of 6 P. ophryomegas $(17 \%, 1)$.

Site of infection: Large intestine.

Additional Costa Rican records: Talamanca rocket frog, Allobates talamancae (Goldberg and Bursey, 2010); Bransford's robber frog, Craugastor bransfordii (Goldberg and Bursey, 2010); Fitzinger's robber frog, Craugastor fitzingeri (Goldberg and Bursey, 2008); black-lined robber frog, Craugastor melanostictus (Goldberg and Bursey, 2008); Noble's robber frog, Craugastor noblei (Bursey and Brooks, 2010a); rain frog, Craugastor ranoides (Goldberg and Bursey, 2008); Golfito robber frog, Craugastor taurus (Goldberg and Bursey, 2008); Underwood's robber frog, Craugastor underwoodi (Goldberg and Bursey, 2008); Túngara frog, Engystomops pustulosus (Bursey and Brooks, 2010a); southern roundgland toad, Incilius coccifer (Bursey and Brooks, 2010a); yellow toad, Incilius luetkenii (Bursey and Brooks, 2010a); Monte Verden Golden toad, Incilius periglenes (Goldberg and Bursey, 2010); southern Gulf Coast toad, Incilius valliceps (Bursey and Brooks, 2010a); moustached frog, Leptodactylus mystacinus (Bursey and Brooks, 2010a); smoky jungle frog, Leptodactyhlus pentadactylus (Bursey and Brooks, 2010a); Turbo white-lipped frog, Leptodacthylus poecilochilus (Bursey and Brooks, 2010a); Forrer's grass frog, Lithobates forreri (Bursey and Goldberg, 2005; Bursey and Brooks, 2010a); Peralta frog, Lithobates taylori (Bursey and Brooks, 2010a); Vaillant's frog, Lithobates vaillanti (Bursey and Brooks, 2010a); Warszewitsch's frog, Lithobates warszewitschii (Bursey and Goldberg, 2007; Bursey and Brooks, 2010a); strawberry poison frog, Oophaga pumilio (Bursey and Brooks, 2010a); Truando toad, Rhaebo haematiticus (Bursey and Brooks, 2010a); cane toad, Rhinella marina (Bursey and Brooks, 2010a); burrowing toad, Rhinophrynus dorsalis (Bursey and Brooks, 2010a); Boulenger's snouted treefrog, Scinax boulengeri (Bursey and Brooks, 2010a); Mexican treefrog, Smilisca baudini (Bursey and Brooks, 2010a); New Granada crossbanded treefrog, Smilisca phaeota (Bursey and Brooks, 2010a); veined treefrog, Trachycephalus venulosus (Bursey and Brooks, 2010a).

Type host and locality: Rhinella marina, Chiapas, Mexico (reported as Bufo marinus, Caballero, 1949).

Other reports: Green toad, Anaxyrus debilis (Goldberg, Bursey, Ramos, 1995); Arizona toad, Anaxyrus microscaphus (Goldberg, Bursey, Malmos, et al., 1996); Sonoran green toad, Anaxyrus retiformis (Goldberg, Bursey, Sullivan, et al., 1996); Woodhouse's toad, Anaxyrus woodhousii (Goldberg, Bursey, Malmos, et al., 1996); polymorphic robber frog, Craugastor rhodopsis (Goldberg, Bursey, SalgadoMaldonado et al., 2002); Great Plains narrowmouth toad, Gastrophryne olivacea (Goldberg et al., 1998a); marbled toad, Incilius marmoreus (Galicia-Guerrero 
et al., 2000); Sinaloa toad, Incilius mazatlanensis (Goldberg and Bursey, 2002a); Lithobates vaillanti (Goldberg, Bursey, Salgado-Maldonado et al., 2002; Paredes-Calderon et al., 2004); Rhinella marina (Goldberg, Bursey, Salgado-Maldonado et al., 2002); Couch's spadefood, Scaphiopus couchii (Goldberg and Bursey, 1991a, 2005b); Smilisca baudini (Goldberg and Bursey, 2002a); Plains spadefoot, Spea bombifrons (Goldberg and Bursey, 2002b); western spadefoot, Spea hammondii (Goldberg and Bursey, 2002b); Great Basin spadefoot, Spea intermontana (Goldberg and Bursey, 2002b); New Mexico spadefoot, Spea multiplicata (Goldberg, Bursey, Ramos, 1995).

Geographic range: Costa Rica (Bursey and Goldberg, 2005); Mexico (Caballero, 1949); U.S.A. (Goldberg et al., 1998a).

Specimen deposited: Chironius grandisquamis, USNPC 103607; Conophis lineatus, USNPC 103608; Porthidium nasutum, USNPC 103609; Porthidium ophryomegas, USNPC 103610; (vials).

\section{Remarks}

This is the first report of $A$. incerta in snakes; previously it was known only from anurans. Species of Chironius, Conophis, and Porthidium are known to feed on frogs and toads (Savage, 2002); thus, the presence of A. incerta in these hosts may be a byproduct of diet rather than an active infection. Chironius grandisquamis, Conophis lineatus, Porthidium nasutum, and Porthidium ophryomegas represent new host records for A. incerta.

\section{Aplectana itzocanensis Bravo Hollis, 1943}

(Syn. Oxysomatium itzocanensis [Bravo Hollis, 1943] Skrjabin, Schikhobalova and Mozgovoi, 1951.)

Hosts and locality: Drymobius margaritiferus (1): UT 02-P-0779, collected 23 October 2002, Area Administrative, Guanacaste Conservation Area; Liophis epinephelus (1): male, UT 03-P-0184, collected 18 February 2003, Sendero Nacho, Guanacaste Conservation Area.

Prevalence and intensity: 1 of 5 D. margaritiferus (20\%, 14); 1 of 1 L. epinephelus $(100 \%, 4)$.

Site of infection: Large intestine.

Additional Costa Rican records: Angel robber frog, Craugastor angelicus (Goldberg and Bursey, 2010); Craugastor fitzingeri (McKenzie, 2007); Fleischmann's robber frog, Craugastor fleischmanni
(Goldberg and Bursey, 2010); evergreen robber frog, Craugastor gollmeri (Goldberg and Bursey, 2008); Craugastor ranoides (Goldberg and Bursey, 2008); Craugastor taurus (Goldberg and Bursey, 2008); Engystomops pustulosus (Goldberg and Bursey, 2010); Incilius coccifer (Bursey and Brooks, 2010a); Incilius luetkenii (Bursey and Brooks, 2010a); Incilius valliceps (Bursey and Brooks, 2010a); Lithobates forreri (Bursey and Goldberg, 2005); Lithobates vaillanti (McKenzie, 2007); Rancho Redondo frog, Lithobates vibicarius (Bursey and Goldberg, 2006a); Lithobates warszewitschii (Bursey and Goldberg, 2007); Rhaebo haematiticus (Bursey and Brooks, 2010a); Rhinella marina (Brenes and Bravo-Hollis, 1959; Bursey and Brooks, 2010a); Scinax boulengeri (Bursey and Brooks, 2010a); Sipurio snouted treefrog, Scinax elaeochrous (Bursey and Brooks, 2010a); Smilisca baudini (Bursey and Brooks, 2010a); Smilisca phaeota (Bursey and Brooks, 2010a); Nicaragua crossbanded treefrog, Smilisca puma (McKenzie, 2007; Bursey and Brooks, 2010a); Trachycephalus venulosus (Bursey and Brooks, 2010a).

Type host and locality: Spea multiplicata, Puebla, Mexico (reported as Scaphiopus multiplicatus, Bravo-Hollis, 1943).

Other reports: Mexican leaf frog, Agalychnis dacnicolor (Goldberg and Bursey, 2002a); western toad, Anaxyrus boreas (Goldberg, Bursey, Hernandez, 1999); Great Plains toad, Anaxyrus cognatus (Goldberg and Bursey, 1991a; Goldberg, Bursey, Ramos, 1995); Anaxyrus debilis (Goldberg, Bursey, Ramos, 1995); little Mexican toad, Anaxyrus kelloggi (Goldberg and Bursey, 2002a); Anaxyrus microscaphus (Goldberg, Bursey, Malmos, et al., 1996); redspotted toad, Anaxyrus punctatus (Goldberg and Bursey, 1991b); Anaxyrus retiformis (Goldberg, Bursey, Sullivan, et al., 1996); Anaxyrus woodhousii (Goldberg, Bursey, Malmos, et al., 1996); smallheaded treefrog, Dendropsophus microcephalus (Goldberg et al., 2002); Gastrophryne olivacea (Goldberg et al., 1998a); Sonoran Desert toad, Incilius alvarius (Goldberg and Bursey, 1991a; Baker, 1985); Incilius mazatlanensis (Goldberg and Bursey, 2002a); Incilius valliceps (Espinola-Novelo and Guillen-Hernandez, 2008); Sabinal frog, Leptodactylus melanonotus (Goldberg and Bursey, 2002a); Northwest Mexico leopard frog, Lithobates magnaocularis (Goldberg and Bursey, 2002a); Pacific chorus frog, Pseudacris regilla (Goldberg, Bursey, Gergus, 2001); Rhinella marina (Bravo Hollis, 1943; Caballero-Deloya, 1974; Espinoza-Jimenez et al., 2007; 
Espinol-Novelo and Guillen-Hernanez, 2008); Scaphiopus couchii (Tinsley, 1990; Goldberg and Bursey, 2005b); blue-spotted Mexican treefrog, Smilisca cyanosticta (Goldberg, Bursey, SalgadoMaldonado, et al., 2002); northern casquehead frog, Smilisca fodiens (Goldberg, Bursey, Galindo, 1999); Spea bombifrons (Goldberg and Bursey, 2002b); Spea multiplicata (Goldberg, Bursey, Ramos, 1995).

Geographic range: Costa Rica (Brenes and Bravo Hollis, 1959); Mexico (Bravo Hollis, 1943); U.S.A. (Baker, 1985).

Specimen deposited: Drymobius margaritiferus, USNPC 103611; Liophis epinephelus USNPC 103612; (vials).

\section{Remarks}

This is the first report of A. itzocanensis in snakes; previously, it was known only from anurans. Drymobius margaritiferus and Liophis epinephelus feed mainly on frogs and toads (Savage, 2002); thus, the presence of A. itzocanensis in these hosts may be a by-product of diet. Drymobius margaritiferus and Liophis epinephelus represent new host records for $A$. itzocanensis.

\section{Cosmocercoides variabilis (Harwood, 1930) Travassos, 1931}

(Syn. Cosmocercodies dukae [Holl, 1928] Travassos, 1931, in part; Oxysomatium variabilis Harwood, 1930; Aplectana americana Walton, 1929; Oxyuris sp. of Waitz, 1961; Oxyuris dubia Leidy, 1856.)

Hosts and locality: Crotalus durissus (1): Female, UT 00-P-0003, collected 23 June 2000 (roadkill), Entrada principal, Guanacaste Conservation Area; Dendrophidion percarinatus (1): UT 03-P-0087, collected 16 January 2003, Rio Negro, Guanacaste Conservation Area.

Prevalence and intensity: 1 of 11 C. durissus (9\%, 4); 1 of 1 D. percarinatus $(100 \%, 2)$.

Site of infection: C. durissus, stomach; D. percarinatus rectum.

Additional Costa Rican records: Red-eyed treefrog, Agalychnis callidryas (Bursey and Brooks, 2010a); Craugastor fitzingeri (Bursey and Brooks, 2010a); Craugastor gollmeri (Goldberg and Bursey, 2008); litter frog, Craugastor megacephalus (Bursey and Brooks, 2010a); Craugastor ranoides (Goldberg and Bursey, 2008); Stejneger's robber frog, Crau- gastor stejnegerianus (Bursey and Brooks, 2010a); Isla Bonita tree frog, Duellmanohyla rufioculus (Bursey and Brooks, 2010a); evergreen toad, Incilius coniferus (Bursey and Brooks, 2010a); Leptodactylus poecilochilus (Bursey and Brooks, 2010a); Lithobates forreri (Bursey and Brooks, 2010a); Lithobates taylori (Bursey and Brooks, 2010a); Lithobates vaillanti (Bursey and Brooks, 2010a); Lithobates warszewitschii (Bursey and Brooks, 2010a); Rhinella marina (Bursey and Brooks, 2010a); copper anole, Anolis cupreus (Bursey and Brooks, 2010b); humble anole, Anolis humilis (Bursey and Brooks, 2010b); lion anole, Anolis lionotus (Bursey and Brooks, 2010b); green basilisk, Basiliscus plumifrons (Bursey and Brooks, 2010b); Cope's alligator lizard, Mesaspis monticola (Bursey and Goldberg, 2006b); spotted coffee snake, Ninia maculata (Goldberg and Bursey, 2006a).

Type host and locality: Gulf Coast toad, Incilius nebulifer, Texas (reported as B. valliceps, Harwood, 1930).

Other reports: Black salamander, Aneides flavipunctatus (Lehmann, 1960); arboreal salamander, Aneides lugubris (Lehmann, 1960); long-toed salamander, Ambystoma macrodactylum (Waitz, 1961); mole salamander, Ambystoma talpoideum (Harwood, 1932); Texas salamander, Ambystoma texanum (Harwood, 1930, 1932); marbled salamander, Dicamptodon ensatus (Lehmann, 1960); northern dusky salamander, Desmognathus fuscus (Odlaug, 1954); large-blotched salamander, Ensatina eschscholtzii (Goldberg et al., 1998b); dark-sided salamander, Eurycea longicauda (McAllister and Bursey, 2004); black-spotted newt, Notophthalmus meridionalis (Harwood, 1930, 1932); red-backed salamander, Plethodon cinereus (Bursey and Schibli, 1995); Sequoyah slimy salamander, Plethodon sequoyah (McAllister and Bursey, 2004); red-bellied newt, Taricha rivularis (Goldberg, Bursey, Dailey, 2009); California newt, Taricha torosus (Ingles, 1936); American toad, Anaxyrus americanus (Vanderburgh and Anderson, 1987a; Joy and Bunten, 1997; Bolek and Coggins, 2000; Yoder and Coggins, 2007); Anaxyrus boreas (Ingles, 1936; Frandsen and Grundmann, 1960; Koller and Gaudin, 1977; Goldberg, Bursey, Hernandez, 1999); Anaxyrus debilis (McAllister et al., 1989); Canadian toad, Anaxyrus hemiophrys (Bursey and Goldberg, 1998); oak toad, Anaxyrus quercicus (Walton, 1938; Hamilton, 1955; Goldberg and Bursey, 1996); southern toad, Anaxyrus terrestris (Harwood, 1932); Anaxyrus wood- 
housii (McAllister et al., 1989); narrow-mouthed toad, Gastrophryne carolinensis (Harwood, 1930, 1932; McAllister and Bursey, 2005); Cope's gray treefrog, Hyla chrysoscelis (Bolek and Coggins, 1998); squirrel treefrog, Hyla squirella (Harwood, 1930, 1932); Incilius nebulifer (Harwood, 1932; McAllister et al., 1989); crawfish frog, Lithobates areolatus (Harwood, 1930, 1932); Plains leopard frog, Lithobates blairi (Goldberg et al., 2000); Carolina gopher frog, Lithobates capito (Walton, 1947); bullfrog, Lithobates catesbeianus (Walton, 1929; Harwood, 1932; Trowbridge and Hefley, 1934; Brandt, 1936; Ingles, 1936; Rankin, 1945; Lehmann, 1965; Campbell, 1968; Hollis, 1972; Rau et al., 1978; Muzzall, 1991; Andrews et al., 1992; Bursey and DeWolf, 1998; McAlpine and Burt, 1998; Goldberg and Bursey, 2002c); green frog, Lithobates clamitans (Harwood, 1932; Odlaug, 1954; Rau et al., 1978; Muzzall, 1991; Bursey and DeWolf, 1998; McAlpine and Burt, 1998); Lithobates magnaocularis (Goldberg and Bursey, 2002a); pickeral frog, Lithobates palustris (Walton, 1929; Harwood, 1930, 1932; Coggins and Sajdak, 1982; McAllister et al., 1995; Bursey and DeWolf, 1998); northern leopard frog, Lithobates pipiens (Odlaug, 1954; Rau et al., 1978; McAlpine and Burt, 1998; Goldberg, Bursey, McKinnell, et al., 2001); southern leopard frog, Lithobates sphenocephala (Harwood, 1930, 1932); wood frog, Lithobates sylvaticus (Harwood, 1930; Odlaug, 1954; Muzzall and Peebles, 1991); spotted chorus frog, Pseudacris clarkii (McAllister, 1991); spring peeper, Pseudacris crucifer (Muzzall and Peebles, 1991); Pseudacris regilla (Lehmann, 1965; Koller and Gaudin, 1977); midland chorus frog, Pseudacris triseriata (Harwood, 1930, 1932; Odlaug, 1954; Bolek and Coggins, 1998); red-legged frog, Rana aurora (Ingles, 1936; Walton, 1947; Lehmann, 1965; Kennedy, 1977); yellow-legged frog, Rana boylei (Bursey et al., 2010); Oregon spotted frog, Rana pretiosa (Lehmann, 1965); Hurter's spadefoot, Scaphiopus hurterii (McAllister et al., 2005); imbricate alligator lizard, Barisia imbricata (Goldberg, Bursey, Camarillo-Rangel, 1999); rough teiid, Echinosaura horrida (Bursey et al., 2007); northern alligator lizard, Elgaria coerulea (Goldberg and Bursey, 1991c); Madrean alligator lizard, Elgaria kingii (Goldberg, Bursey, Cheam, 1999); San Lucan alligator lizard, Elgaria paucicarinata (Goldberg, Bursey, Beaman, 2004); Texas alligator lizard, Gerrhonotus liocephalus (Goldberg, Bursey, Camarillo-Rangel, 1999); Mediterranean house gecko,
Hemidactylus turcicus (Criscione and Font, 2001); slender glass lizard, Ophisaurus attenuatus (Harwood, 1930, 1932); five-lined skink, Plestiodon fasciatus (Harwood, 1932); northern fence lizard, Sceloporus undulatus (Goldberg, Bursey, McAllister, 1995); ground skink, Scincella lateralis (Harwood, 1930, 1932; Goldberg, Bursey, McAllister, 1995); cottonmouth, Agkistrodon piscivorus (Fontenot and Font, 1996); eastern hognose snake, Heterodon platyrhinos (Harwood, 1930, 1932); harlequin coral snake, Micrurus fulvius (Harwood, 1930, 1932); brown snake, Storeria dekayi (Harwood, 1930, 1932; Rau et al., 1978; Rau and Gordon, 1980); three-toed box turtle, Terrapene carolina (Harwood, 1930, 1932; Rausch, 1947); ornate box turtle, Terrapene ornata (Harwood, 1930, 1932).

Geographic range: Canada (Vanderburgh and Anderson, 1987a); Costa Rica (Goldberg and Bursey, 2006a); Mexico (Goldberg, Bursey, Camarillo-Rangel, 1999); Panama (Bursey et al., 2007); U.S.A. (Harwood, 1930).

Specimen deposited: Crotalus durissus, USNPC 103613; Dendrophidion percarinatus USNPC 103614; (vials).

\section{Remarks}

Some uncertainty exists for hosts of the 2 American species of Cosmocercoides: Cosmocercoides dukae, originally Cosmocerca dukae Holl, 1928 and Cosmocercoides variabilis, originally Oxysomatium variabiliis Harwood, 1930. Wilkie (1930) established the genus Cosmocercoides, and Travassos (1931) included both C. dukae and C. variabilis in his monograph on the Cosmocercidae. Ogren $(1953,1959)$ considered C. variabilis a synonym of the molluscian parasite $C$. dukae and presumed that amphibians acquired $C$. dukae by ingesting infected molluscs. Vanderburgh and Anderson (1987b) demonstrated that these 2 species were distinct. The major difference in the 2 species is the number of rosette papillae of the male: $C$. dukae with 12 pairs; $C$. variabilis with 14 to 20 pairs. Two males and 2 females were collected in this study and, because the males exhibited 16 pairs of rosette papillae, they are assigned to $C$. variabilis. Ingles (1936) reported C. dukae from Taricha torosa, Rana aurora, and Bufo boreas from California but illustrated 16 papillae and, for this reason, his specimens were referred to $C$. variabilis. Anderson (2000) refers all infections of $C$. dukae in toads to $C$. 
variabilis. Cosmocercoides variabilis has been previously reported from snakes (Harwood, 1930, 1932; Rau et al., 1978; Rau and Gordon, 1980; Fontenot and Font, 1996). Crotalus durissus and Dendrophidion percarinatus represent new host records for $C$. variabilis.

\section{Kathlaniidae (Lane, 1914, subfam.) Travassos, 1918 Cruziinae (Travassos 1917, fam.) Ortlepp, 1924 \\ Cruzia rudolphi Ruiz, 1947}

Hosts and locality: Sibon nebulata (2): UT 01-P0403 collected 23 June 2001; UT 01-P-0420, 25 June 2001, Buenos Aires, Guanacaste Conservation Area.

Prevalence and intensity: 2 of 2 S. nebulata (100\%, 13, 36, respectively).

Site of infection: Large intestine.

Additional Costa Rican records: None.

Type host and locality: Aesculapian false coralsnake, Erythrolamprus aesculapii, Brazil (Ruiz, 1947).

Other reports: Catesby's snail-eater, Dipsas catesbyi, (McAllister et al., 2010); Neotropical snail eater, Dipsas indica, (McAllister et al., 2010).

Geographic range: Brazil (Ruiz, 1947); Costa Rica (this study); Ecuador (McAllister et al., 2010).

Specimen deposited: Sibon neubulatus, USNPC 103615, 103616; (vials).

\section{Remarks}

Sibon neubulatus represents a new host record for C. rudolphi; Costa Rica is a new locality record.

\section{Seuratoidea \\ Seuratidae (Hall, 1916) Railliet, 1916 Skrjabinelaziinae Chabaud, Campana-Rouget and Brygoo, 1959 Skrjabinelazia intermedia (Freitas, 1940) Chabaud, 1973}

(Syn. Salobrella intermedia, Freitas, 1940.)

Hosts and locality: Oxybelis fulgidus (1), male, UT 04-P-0431, collected 15 June 2004, Playa Junquillal, Guanacaste Conservation Area.

Prevalence and intensity: 1 of 3 O. fulgidus (33\%, $1)$.
Site of infection: Intestine.

Additional Costa Rican records: Lichen anole, Norops pentaprion (Goldberg and Bursey, 2004b).

Type host and locality: Tropidurus spinulosus, Brazil (Freitas, 1940)

Other reports: Anolis punctatus (Bursey et al., 2005); Cnemidophorus nativo (Menezes et al., 2004); Stenocercus caducus (Avila, Souza and da Silva, 2010); Tropidurus guarani (Bursey and Goldberg, 2004); Tropidurus spinulosus (Vincente, 1981); Tropidurus torquatus (Vicente, 1981).

Geographic range: Brazil (Freitas, 1940); Costa Rica (this study); Paraguay (Bursey and Goldberg, 2004); Peru (Bursey et al., 2005).

Specimen deposited: Oxybelis fulgidus, USNPC 103617; (vials).

\section{Remarks}

Skrjabinelazia intermedia was previously known only from lizards (see host list above). Oybelis fulgidus is known to feed on lizards (Savage, 2002); thus, this occurrence may be a by-product of diet rather than an active infection. Oxybelis fulgidus represents a new host record for $S$. intermedia.

\section{Ascaridoidea \\ Ascarididae Baird, 1853 \\ Ascaridinae Baird, 1853 \\ Hexametra boddaertii (Baird, 1860) \\ Sprent, 1978}

(Syn. Ascaris boddaertii Baird, 1860; Ascaris quadrangularis Schneider, 1866, in part; Polydelphis hexauterina Skrjabin, 1916; Hexametra quadricornis [Wedl, 1861] sensu Araujo, 1969; Polydelphis quadrangularis of Araujo, 1969).

Hosts and locality: Agkistrodon bilineatus (1), male, UT 03-P-0399, collected 25 May 2003, Caretera Interamericana, Guanacaste Conservation Area; Crotalus durissus (1), UT 02-P-1001, collected 28 December 2002, Area Administrativa, Guanacaste Conservation Area; Porthidium ophryomegas (1), female, UT 04-P-0438, collected 14 June 2004, Centro, Guanacaste Conservation Area; Spilotes pullatus (1), UT 03-P-0154, collected 30 January 2003, on road to Santa Rosa, Guanacaste Conservation Area; Trimorphodon biscutatus (1), UT 03-P- 
0329, collected 10 May 2003, Area Administrativa, Guanacaste Conservation Area.

Prevalence and intensity: 1 of 2 A. bilineatus $(50 \%, 2) ; 1$ of 11 C. durissus $(9 \%, 2) ; 1$ of $1 P$. ophryomegas $(100 \%, 1) ; 1$ of 2 S. pullatus $(50 \%$, 53); 1 of 7 T. biscutatus $(14 \%, 2)$.

Site of infection: Intestine, body cavity.

Additional Costa Rican records: Crotalus durissus (Bowman, 1984); Micrurus nigrocinctus (Goldberg and Bursey, 2004a).

Type host and locality: Mastigodryas boddaerti, West Indies (Baird, 1860).

Other reports: Bothrops sp. (Skrjabin, 1916; Sprent, 1978); Crotalus adamanteus (Sprent, 1978); Crotalus atrox (Sprent, 1978; Goldberg et al., 2002); Crotalus basiliscus (Goldberg, Bursey, Beaman et al., 2006); Crotalus cerastes (Bursey et al., 1995); Crotalus confluentus (Sprent, 1978); Crotalus durissus (Araujo, 1969; Sprent, 1978; Vicente et al., 1993); Crotalus viridus (Bowman, 1984); Baron's racer, Phylodryas baroni (Hartdegen and Gamble, 2002); Philodryas schottii (Sprent, 1978); Pseudoboa trigeminal (Sprent, 1978) Sistrurus catenatus (Goldberg, Bursey, Holycross, 2001); immature specimen have been reported in the lizards, Cnemidophorus ocellifer (Dias et al., 2005); Mabuya agilis (Vrcibradic et al., 2002); Mabuya macrorhyunchia (Vrcibradic et al., 2001, 2002).

Geographic range: Brazil (Araujo, 1969); Costa Rica (Bowman, 1984); Mexico (Goldberg, Bursey, Beaman et al., 2006); Paraguay (Skrjabin, 1916); West Indies (Baird, 1860); United States: California (Bursey et al., 1995); New Mexico (Goldberg, Bursey, Holycross, 2001).

Specimen deposited: Agkistrodon bilineatus, USNPC 103618; Crotalus durissus, USNPC 103619; Porthidium ophryomegas, USNPC 103620; Trimorphodon biscutatus, USNPC 103621; (vials).

\section{Remarks}

Agkistrodon bilineatus, Porthidium ophryomegas, Spilotes pullatus, and Trimorphodon biscutatus represent new host records for $H$. boddaertii.

\section{Ophidascaris arndti Sprehn, 1929}

(Syn. Ascaris quadriangularis Schneider, 1866 in part; Ophidascaris travassosi Vaz, 1938; Ophidascaris sprenti Araujo, 1969).
Hosts and locality: Crotalus durissus (3): UT 02P-1001, collected 28 December 2002, Area Administrativa, Guanacaste Conservation Area; female, roadkill, UT 04-P-0001, collected 1 June 2004, Paracela Principe, Guanacaste Conservation Area; juvenile, UT 04-P-0461, collected 5 November 2004, Area Administrativa, Guanacaste Conservation Area.

Prevalence and intensity: 3 of 11 C. durissus (27\%, 5, 1, 2, respectively).

Site of infection: Stomach, mesentery.

Additional Costa Rican records: None.

Type host and locality: Brazilian snake, Berlin Aquarium, Berlin, Germany, identified as Lachesis lanceolatus in Sprehn, (1929); currently Martinique lancehead, Bothrops lanceolatus (Lacépède, 1789). Perhaps a misidentified host, Lazell (1964) considers $B$. lanceolatus to be restricted to Martinique.

Other reports: Bothrops atrox (Vicente et al., 1993); Bothrops cotiara (Araujo, 1969); Crotalus durissus (Araujo, 1969; Vaz, 1938); Micrurus lemniscatus (McAllister et al., 2010); Porthidium hespere (Goldberg et al., 2008).

Geographic range: Brazil (Schneider, 1866); Costa Rica (this study); Ecuador (McAllister et al., 2010); Mexico (Goldberg et al., 2008).

Specimen deposited: Crotalus durissus, USNPC 103622-103624; (vials).

\section{Remarks}

Costa Rica is a new locality record for $O$. arndti.

\section{Ophidascaris sicki Freitas, 1951}

(Syn. Ophidascaris ardti Sprehn, 1929 sensu Freitas, 1955; Ophidascaris cretiorum Freitas, 1968).

Hosts and locality: Xenodon rabdocephalus (1): UT 02-P-0870, collected 13 November 2002, on road to Santa Rosa, Guanacaste Conservation Area.

Prevalence and intensity: 1 of $1 X$. rabdocephalus $(100 \%, 1)$.

Site of infection: Intestine.

Additional Costa Rican records: None.

Type host and locality: Waglerophis (as Xenodon) merremii, Brazil (Freitas, 1951).

Other reports: Leimadophis poecilogyrus (Sprent, 1988); Micrurus frontalis (Sprent, 1988); Philodryas 
patagoniensis (Sprent, 1988); Pseudoboa cloelia (Sprent, 1988); Waglerophis merremii (Freitas, 1955); Xenodon neuwiedii (Sprent, 1988); Xenodon severus (Freitas, 1951; Vicente et al., 1993).

Geographic range: Brazil (Freitas, 1951); Costa Rica (this study).

Specimen deposited: Xenodon rabdocephalus, USNPC 103625; (vials).

\section{Remarks}

Xenodon rabdocephalus represents a new host record for $O$. sicki. Costa Rica is a new locality record.

\section{Travassosascaris araujoi Sprent, 1978}

(Syn. Polydelphis quadrangularis [Schneider, 1866] sensu Araujo, 1969.)

Hosts and locality: Masticophis mentovarius (1): UT 03-P-0361, collected 23 May 2003, Interamerican Highway, Guanacaste Conservation Area; Xenodon rabdocephalus (1): UT 02-P-0870, collected 13 November 2002, road to Santa Rosa, Guanacaste Conservation Area.

Prevalence and intensity: 1 of $1 M$. mentovarius $(100 \%, 4) ; 1$ of $1 X$. rabdocephalus $(100 \%, 1)$.

Site of infection: Intestine.

Additional Costa Rican records: None.

Type host and locality: Crotalus durissus terrificus, Brazil (Araujo, 1969).

Other reports: None.

Geographic range: Brazil (Araujo, 1969); Central America (Sprent, 1978); Costa Rica (this study).

Specimen deposited: Masticophis mentovarius, USNPC 103626; Xenodon rabdocephalus USNPC 103627; (vials).

\section{Remarks}

Masticophis mentovarius and Xenodon rabdocephalus represent new host records for $O$. sicki. Costa Rica is a new locality record.

\section{Immature ascaridids (unidentified)}

Hosts and locality: Chironius grandisquamis (1): male, UT 03-P-0241, collected 14 March 2003, Rio Mena, Guanacaste Conservation Area; Imantodes inornatus (1): male, UT 03-P-0442, collected 25 June
2003, Sendero Laguna, Guanacaste Conservation Area; Leptodrymus pulcherrimus (1): female, roadkill, UT 04-P-0006, collected 3 June 2004, Santa Elena, Guanacaste Conservation Area; Oxybelis aeneus (1): female, UT 02-P-0908, collected 19 November 2002; Laguna Los Jicaros, Guanacaste Conservation Area; Trimorphodon biscutatus (1): UT 03-P-0329, collected 10 May 2003, Area Administrativa, Guanacaste Conservation Area

Prevalence and intensity: 1 of $1 C$. grandisquamis (100\%, 10); 1 of 1 I. inornatus $(100 \%, 1) ; 1$ of $1 \mathrm{~L}$. pulcherrimus $(100 \%, 1) ; 1$ of 5 O. aeneus $(20 \%, 1) ; 1$ of $7 \mathrm{~T}$. biscutatus $(14 \%, 4)$.

Site of infection: Intestine, mesentery.

Additional Costa Rican records: Anurans: Incilius coccifer, Incilius luetkenii, Leptodactylus pentadactylus, Lithobates forreri (Bursey and Brooks, 2010a).

Other reports: Microlophus occipitalis (Peru; Goldberg and Bursey, 2009c); Drymobius margaritiferus (Nicaragua; Goldberg and Bursey, 2005a).

Specimen deposited: Chironius grandisquamis, USNPC 103628; Imantodes inornatus, USNPC 103629; Leptodrymus pulcherrimus, USNPC 103630; Oxybelis aeneus, USNPC 103631; Trimorphodon biscutatus, USNPC 103632; (vials).

\section{Remarks}

The structure of the esophagus would allow assignment to the Ascaridinae.

\section{Toxocarinae Porrocaecum sp. (immature)}

Hosts and locality: Dendrophidion percarinatus (1): (UT 03-P-0087) collected 16 January 2003, Rio Negro, Guanacaste Conservation Area; Oxybelis brevirostris (1): male (UT 03-P-0384) collected 23 May 2003, Quebrada abajo de los Naranjales, Guanacaste Conservation Area.

Prevalence and intensity: 1 of $1 D$. percarinatum $(100 \%, 11) ; 1$ of 2 O. brevirostris $(50 \%, 1)$.

Site of infection: Stomach.

Additional Costa Rican records: Rusty head snake, Amastridium veliferum (Goldberg and Bursey, 2004a); sipo, Chironius carinatus (Goldberg and Bursey, 2004a); yellowbelly snake, Coniophanes fissidens (Goldberg and Bursey, 2007a); Dendrophidion pericarinatum (Goldberg and Bursey, 2004a); barred forest racer, Dendrophidion vinitor (Goldberg 
and Bursey, 2004a); false coral snake, Erytolampus bizona (Goldberg and Bursey, 2004a); western tree snake, Imantodes inorntus (Goldberg and Bursey, 2009a); northern cat-eyed snake, Leptodeira septentrionalis (Goldberg and Bursey, 2009b); yellowbellied racer, Liophis epinephalis (Goldberg and Bursey, 2004a); Central American coral snake, Micrurus nigrocinctus (Goldberg and Bursey, 2004a); Oxybelis brevirostris (Goldberg and Bursey, 2004a); Pliocercus euryzonus (Goldberg and Bursey, 2007a); adorned graceful brown snake, Rhadinea decorata (Goldberg and Bursey, 2007a); leaf litter gecko, Lepidoblepharis xanthostigma (Goldberg and Bursey, 2008); Craugastor fitzingeri (Goldberg and Bursey, 2008); Lithobates taylori (Goldberg and Bursey, 2007b); Lithobates warszewitschii (Bursey and Goldberg, 2007); Rio San Juan robber frog, Pristimantis ridens (Goldberg and Bursey, 2008).

Type host and locality: Adults are parasites of birds.

Other reports: Salmon-bellied racer, Mastigodryas melanolomus (Goldberg and Bursey, 2006b); West Coast garter snake, Thamnophis valida (Goldberg and Bursey, 2004c); Auber's ameiva, Ameiva auberi (Barus and Coy Otero, 1969; Coy Otero, 1970; Coy Otero and Barus, 1979); Allison's anole, Anolis allisoni (Coy Otero and Barus, 1979); Bueycito anole, Anolis allogus (Coy Otero and Barus, 1979); Monte Verde anole, Anolis alutaceus (Coy Otero and Barus, 1979); bay anole, Anolis argillaceus (Coy Otero and Barus, 1979); Baracoa anole, Anolis baracoae (Coy Otero and Barus, 1979); western cliff anole, Anolis bartschi (Coy Otero and Barus, 1979); Heradura anole, Anolis bremeri (Coy Otero and Barus, 1979); Grand Cayman anole, Anolis conspersus (Goldberg, Bursey, Cheam, 1995); crested anole, Anolis cristatellus (Goldberg et al., 1998c); knight anole, Anolis equestris (Barus and Coy Otero, 1968, 1969; Coy Otero, 1970); Etheridge's anole, Anolis etheridgei (Goldberg et al., 1998d); Habana anole, Anolis homolechis (Barus and Coy Otero, 1969; Coy Otero, 1970; Coy Otero and Barus, 1979); Cubitas anole, Anolis jubar (Coy Otero and Barus, 1979); San Lucian anole, Anolis luciae (Goldberg et al., 1997a); cave anole, Anolis lucius (Coy Otero and Barus, 1979); white-throated anole, Anolis luteogularis (Coy Otero and Barus, 1979); snakescale anole, Anolis ophiolepis (Coy Otero and Barus, 1979); Oriente bearded anole, Anolis porcus (Coy Otero and Barus, 1979); redbeard anole, Anolis rubribarbatus (Coy Otero and Barus, 1979); brown anole, Anolis sagrei
(Coy Otero and Barus, 1979; Goldberg et al., 1994a); longnose leopard lizard, Gambelia wislizeni (McAllister and Bursey, 2007); house gekko, Hemidactylus mabouia (Coy Otero and Barus, 1979); northern curly-tailed lizard, Leiocephalus carinatus (Barus and Coy Otero, 1969; Coy Otero, 1970; Coy Otero and Barus, 1979; Goldberg, Bursey and Cheam, 1995); Cuban curly-tailed lizard, Leiocephalus cubensis (Barus and Coy Otero, 1969; Coy Otero, 1970; Coy Otero and Barus, 1979); Monte Verde curlytail lizard, Leiocephalus macropus (Barus and Coy Otero, 1969; Coy Otero, 1970; Coy Otero and Barus, 1979); mountain curlytail lizard Leiocephalus raviceps (Coy Otero and Barus, 1979); Cabo corrientes curlytail lizard, Leiocephalus stictigaster (Coy Otero and Barus, 1979); American wall gecko, Tarentola americana (Coy Otero and Barus, 1979); Condoto stubfoot toad, Atelopus spurrelli (Goldberg and Bursey, 2003); polymorphic robber frog, Craugastor rhodopis (Goldberg et al., 2002); El Yungue robber frog, Eleutherodactyus acmonis (Coy Otero and Ventosa, 1984); rainfrog, Eleutherodactylus amplinympha (Goldberg et al., 1998); Atkins' robber frog, Eleutherodactylus atkinsi (Coy Otero and Ventosa, 1984); Juventud robber frog, Eleutherodactlyus cuneatus (Barus, 1973); black whiskered frog, Eleutherodactylus dimidiatus (Barus, 1973; Coy Otero and Ventosa, 1984); Gundlach's robber frog, Eleutherodactylus gundlachi (Coy Otero and Ventosa, 1984); Klinikowski's robber frog, Eleutherodactylus klinikowskii Coy Otero and Ventosa, 1984); Martinique robber frog Eleutherodactylus martinicensis (Moravec and Kaiser, 1995; Goldberg et al., 1998); greenhouse frog, Eleutherodactylus planirostris (Coy Otero and Ventosa, 1984); Ronald's robber frog, Eleutherodactylus ronaldi (Coy Otero and Ventosa, 1984); Symington's robber frog, Eleutherodactyus symingtoni (Coy Otero and Ventosa, 1984); Thomas' robber frog, Eleutherodactylus thomasi (Coy Otero and Ventosa, 1984); Varley's robber frog, Eleutherodactylus varleyi (Coy Otero and Ventosa, 1984); Zeus' robber frog, Eleutherodactylus zeus (Coy Otero and Ventosa, 1984); Sabinal frog, Leptodactylus melanonotus (Goldberg et al., 2002); bullfrog, Lithobates catesbeianus (Coy Otero and Ventosa, 1984); Cuban treefrog, Osteopilus septentrionalis (Coy Otero and Ventosa, 1984); Cuban long-nosed toad, Peltophryne longinasus (Coy Otero and Ventosa, 1984); Tschudi's Caribbean toad, Peltophryne peltocephala (Coy Otero and Ventosa, 1984); Cuban spotted toad, Peltophryne taladai (Barus, 1973); Surinam toad, Pipa pipa (Bursey et al., 2001); 
Charlotteville rain frog, Pristimantis charlottevillensis (Goldberg et al., 1998); Grenada rain frog, Pristimantis euphronides (reported as Eleutherodactylus euphronides, Moravec and Kaiser, 1995).

Geographic range: The genus is cosmopolitan. Immature forms listed above are all Western Hemisphere: Bahamas (Goldberg et al., 1994); British Virgin Islands (Goldberg et al., 1988a); Cayman Islands (Goldberg, Bursey and Cheam, 1995); Colombia (Goldberg and Bursey, 2003); Cuba (Barus and Coy Otero, 1968); Dominica (Moravec and Kaiser, 1995); Grenada (Moravec and Kaiser, 1995); Guadeloupe (Goldberg et al., 1998); Hispaniola (Goldberg et al., 1998d); Marie Galante (Moravec and Kaiser 1995); Mexico (Goldberg et al., 2002); Peru (Bursey et al., 2001); St. Lucia (Goldberg et al., 1997a); Tobago (Goldberg et al., 1998).

Specimen deposited: Dendrophidion percarinatus USNPC 103633; Oxybelis brevirostris, USNPC 103634; (vials).

\section{Remarks}

Eggs passed with feces embryonate to first stage larvae, then molt to second stage; eggs must be ingested by earthworms, in which hatching occurs, and the second stage larvae develop to third stage. Definitive hosts may acquire infection by ingesting infected earthworms directly or by eating infected vermivores (Anderson, 2000). All hosts listed above should be considered paratenic hosts.

\section{Anisakinae Railliet and Henry, 1912 Terranova caballeroi Barus and Coy Otero, 1966}

Hosts and locality: Erythrolamprus mimus (1): female, UT 03-P-0257, collected 18 March 2003, Estacion, Guanacaste Conservation Area.

Prevalence and intensity: 1 of 2 E. minus $(50 \%, 4)$.

Site of infection: Stomach.

Additional Costa Rican records: Liophis epinephalis (Goldberg and Bursey, 2004a).

Type host and locality: Alsophis canthigerus, Cuba (Barus and Coy Otero, 1966).

Other reports: Alsophis canthigerus (Barus and Coy Otero, 1978); Ancistrodon piscivorus (Sprent, 1979; Fontenot and Font, 1996); Coluber constrictor (Sprent, 1979); eastern indigo snake, Drymarchon corais (Foster et al., 2000); Heterodon platyrhinos (Sprent, 1979); Nerodia cyclopon (Sprent, 1979;
Fontenot and Font, 1996); Nerodia fasciata (Fontenot and Font, 1996); Nerodia rhombifera (Fontenot and Font, 1996); Nerodia taxispilota (Sprent, 1979); Nerodia sipedon (Sprent, 1979).

Geographic range: Costa Rica (Goldberg and Bursey, 2004a); Cuba (Barus and Coy Otero, 1966); Florida and Louisiana, U.S.A. (Sprent, 1979).

Specimen deposited: Erythrolamprus mimus, USNPC 103635; (vials).

\section{Remarks}

Erythrolamprus mimus represents a new host record for $T$ caballeroi.

\section{SPIRURIDA \\ Physalopteroidea \\ Physalopteridae (Railliet, 1893) Leiper, 1908 Physalopterinae Railliet, 1893 Abbreviata costaricae n. sp.}

Hosts and locality: Agkistrodon bilineatus (1): male, UT 03-P-0399, collected 25 May 2003, Interamerican Highway, Guanacaste Conservation Area; Masticophis mentovarius (1): UT 03-P-0361, collected 23 May 2003, Interamerican Highway, Guanacaste Conservation Area.

Prevalence and intensity: 1 of 2 A. bilineatus (50\%, 15); 1 of $1 \mathrm{M}$. mentovarius $(100 \%, 1)$.

Site: Stomach.

Additional Costa Rican records: None.

Type host and locality: Agkistrodon bilineatus, Costa Rica (this study).

Other reports: None.

Geographic range: Costa Rica (this study).

Specimen deposited: Masticophis mentovarius, USNPC 103606; (vial).

\section{Remarks}

See Discussion section above.

\section{Physaloptera retusa Rudolphi, 1819}

(Syn. Spiroptera retusa Dujardin 1845; Physaloptera mucronata Leidy, 1956; Physaloptera largada Sprehn, 1932.)

Hosts and locality: Drymobius margaritiferus (1): UT 02-P-0779, collected 23 October 2002, Area 
administrative, Guanacaste Conservation Area; Oxybelis fulgidus (1), male, UT 04-P-0431, collected 15 June 2004, Playa Junquillal, Guanacaste Conservation Area.

Prevalence and intensity: 1 of 5 D. margaritiferus $(20 \%, 1) ; 1$ of 3 O. fulgidus $(33 \%, 1)$.

Site: Large intestine.

Additional Costa Rican records: Anolis cupreus (Goldberg and Bursey, 2004d; Bursey and Brooks, 2010b); Anolis humilis (Bursey and Brooks, 2010b); Anolis lionotus (Bursey and Brooks, 2010b); common basilisk, Basiliscus basiliscus (Bursey and Brooks, 2010b); yellow-headed gecko, Gonatodes albogularis (Bursey and Brooks, 2010b); common house gecko, Hemidactylus frenatus (Bursey and Brooks, 2010b); rosebelly lizard, Sceloporus variabilis (Bursey and Brooks, 2010b).

Type host and locality: Golden tegu, Tupnambis teguixin, Brazil (Rudolphi, 1819).

Other reports: Common lesser toad, Rhinella granulosus (Goncalves et al., 2002); South American common toad, Rhinella margaritifera (Goncalves et al., 2002); giant ameiva, Ameiva ameiva (Poinar and Vaucher, 1972; Cristofaro et al., 1976; Ribas et al., 1998a; Bursey et al., 2005; Bursey et al., 2007); Middle American ameiva, Ameiva festiva (Goldberg and Bursey, 2009d); rainbow ameiva, Ameiva undulata (Caballero, 1951); red worm lizard, Amphisbaena alba (Molin, 1860); brown-eared anole, Anolis fuscoauratus (Goldberg, Bursey, Vitt, 2006); Amazon green anole, Anolis punctatus (Bursey et al., 2005); canyon spotted whiptail, Aspidoscelis burti (Goldberg and Bursey, 1989a); imbricate alligator lizard, Barisia imbricata (Goldberg, Bursey, Camarillo-Rangel, 1999); zebratail lizard, Callisaurus draconoides (Telford, 1970); sand dune lizard, Cnemidophorus abaetensis (Dias et al., 2005); rainbow whiptail, Cnemidophorus lemniscatus (Caballero and Vogelsang, 1947; Diaz-Ungria, 1964; Diaz-Ungria and Gallardo, 1968); green tail lizard, Cnemidophorus littoralis (Vrcibradic et al., 2000); Laurent's whiptail, Cnemidophorus murinus (Specian and Whittaker, 1980); no common name, Cnemidophorus nativo (Menezes et al., 2004): Spix's whiptail, Cnemidophorus ocellifer (Ribas et al., 1995); southern alligator lizard, Elgaria multicarinata (Telford, 1970); San Lucan alligator lizard, Elgaria paucicarinata (Goldberg, Bursey, Beaman, 2004); western skink, Eumeces skiltonianus (Telford, 1970); longnose leopard lizard, Gambelia wislizenii (Telford,
1970); Texas alligator lizard, Gerrhonotus liocephalus (Goldberg, Bursey, Camarillo-Rangel, 1999); yellow headed gecko, Gonatodes albogularis (Bursey et al., 2007); green iguana, Iguana iguana (DiazUngria and Gallardo, 1968; Bursey et al., 2007); no common name, Kentropyx altamazonicus (Bursey et al., 2005); striped forest whiptail, Kentropyx calcarata (Goldberg et al., 2007; Avila and da Silva, 2009); forest whiptail, Kentropyx pelviceps (Bursey et al., 2005); Bell's anole, Leiosaurus belli (Goldberg, Bursey, Morando, 2004); no common name, Leiosaurus catamarcensis (Goldberg, Bursey, Morando, 2004); no common name, Leposoma rugiceps (Bursey et al., 2007); Liolaemus boulengeri (O'Grady and Dearing, 2006); Liolaemus darwinii (O'Grady and Dearing, 2006); Liolaemus koslowskyi (O'Grady and Dearing, 2006); Lutz's tree iguana, Liolaemus lutzae (Rocha, 1995); no common name, Liolaemus nequensis (Goldberg et al., 2004); Liolaemus umbrifer (O'Grady and Dearing, 2006); no common name, Mabuya agilis (Ribas et al., 1998b); twostriped mabuya, Mabuya bistriata (Molin, 1860; Bursey et al., 2005); Paraguay mabuya, Mabuya dorsivittata (Rocha et al., 2003); Mesaspis monticola (Bursey and Goldberg, 2006b); Microlophus koepckeorum (Goldberg and Bursey, 2009c); Microlophus peruvianus (Goldberg and Bursey, 2009c); Microlophus stolzmanni (Goldberg and Bursey, 2009c); Microlophus tigris (Goldberg and Bursey, 2009c); tree runner, Plica plica (Bursey et al., 2005; Goldberg, Bursey, Vitt, 2009); blue-lipped tree lizard, Plica umbra (Bursey et al., 2005; Goldberg, Bursey, Vitt, 2009); Polychrus gutturosus (Bursey et al., 2007); common stream lizard, Potamites ecpleopus (Goldberg et al., 2007); Bocourt's spiny lizard, Sceloporus acanthinus (Caballero, 1951); Clark's spiny lizard, Sceloporus clarkii (Goldberg et al., 1994b); blue spiny lizard, Sceloporus cyanogenys (Goldberg, Bursey, McAllister, 1995); emerald spiny lizard, Sceloporus formosus (Goldberg et al., 2003); sagebrush lizard, Sceloporus graciosus (Woodbury, 1934; Goldberg and Bursey, 1989b; Goldberg et al., 1997b); graphix spiny lizard, Sceloporus grammicus (Goldberg et al., 2003); Yarrow's spiny lizard, Sceloporus jarrovii (Goldberg and Bursey, 1990; Bursey and Goldberg, 1991, 1994; Goldberg, Bursey, McAllister, 1995; Goldberg, Bursey, Bezy, 1996); desert spiny lizard, Sceloporus magister (Pearce and Tanner, 1973; Walker and Matthias, 1973; Goldberg et al., 1994b; Goldberg, Bursey, McAllister, 1995); canyon lizard, Sceloporus merriami (Goldberg, Bursey, McAllister, 1995); southern crevice lizard, Scelo- 
porus mucronatus (Goldberg et al., 2003); western fence lizard, Sceloporus occidentalis (Grundmann, 1959; Telford, 1970; Pearce and Tanner, 1973; Goldberg et al., 1998e); Texas spiny lizard, Sceloporus olivaceus (Goldberg Bursey, McAllister, 1995); granite spiny lizard, Sceloporus orcutti (Telford, 1970); bluebelly lizard, Sceloporus parvus (Goldberg et al., 2003); crevice spiny lizard, Sceloporus poinsettii (Goldberg et al., 1993; Goldberg Bursey, McAllister, 1995); rough-scaled lizard, Sceloporus serrifer (Goldberg Bursey, McAllister, 1995); crevice swift, Sceloporus torquatus (Goldberg et al., 2003); eastern fence lizard, Sceloporus undulatus (Morgan, 1943; Pearce and Tanner, 1973; Goldberg et al., 1994b; Goldberg, Bursey, McAllister, 1995); Sceloporus variabilis (Goldberg, Bursey, McAllister, 1995; Goldberg et al., 2003); striped plateau lizard, Sceloporus virgatus (Goldberg et al., 1994b); rose whorltail iguana, Stenocercus roseiventris (Bursey et al., 2005); turniptail gecko, Thecadactylus rapicauda (Bursey et al., 2005); black lava lizard, Tropidurus melanopleurus (Roca, 1997); spiny lava lizard, Torpidurus spinulosus (Vicente, 1981); Amazon lava lizard, Tropidurus torquatus (Vicente and Santos, 1967; Cristofaro et al., 1976; Vicente, 1981; Ribas et al., 1998a; Vrcibradic et al., 2000); no common name, Tupinambis longilineus (Avila, dos Anjos, Silva et al., 2010); black spotted tegu, Tupinambis nigropunctatus (Morgan, 1943); red tegu, Tupinambis rufescens (Sprehn, 1932); Tupinambis teguixin (Molin, 1860; Ortlepp, 1922; Lent and Freitas, 1948; SchuurmansStekhoven, 1950); thornytail iguana, Uracentron flaviceps (Goldberg and Bursey, 2007c); side-blotched lizard, Uta stansburiana (Telford, 1970).

Geographic range: Argentina (Goldberg, Bursey, Morando, 2004); Brazil (Rudolphi, 1819); Colombia (Goldberg and Bursey, 2009d); Costa Rica (Goldberg and Bursey, 2004a); Mexico (Caballero, 1951); Netherlands Antillels (Specian and Whittaker, 1980); Panama (Bursey, Goldberg, Telford, 2007); Peru (Bursey et al., 2005); Venezula (Diaz-Ungaria and Gallardo, 1968); U.S.A. (Grundmann, 1959).

Specimen deposited: Drymobius margaritiferus, USNPC 103636; Oxybelis fulgidus, USNPC 103637; (vials).

\section{Remarks}

Seven species of Physaloptera have been described from reptiles of the Americas, namely $P$. abjecta Leidy, 1856, $P$. liophis Vincente and Santos 1974, P. lutzi Cristofaro, Guimaraes and Rodrigues,
1976, P. monodens Molin, 1860, P. obtusissima Molin, 1860, P. retusa, and $P$. squamatae Harwood, 1932. Physaloptera monodens is considered a synonym of $P$. obtusissima by Morgan (1943). The 6 species are separated by the position of the vulva or based on the shape of the spicule and pattern of caudal sessile papillae. The vulva is near the anus in $P$. lutzi; in the other 5 species, it occurs in the first third of the body. Physaloptera retusa posseses a bent right spicule; in the remaining 4 species, the spicule is not curved. Physaloptera abjecta has 3 pairs of postcloacal sessile papillae; the remaining 3 species have 5 pairs. In $P$. liophis, the first 2 pairs of postcloacal papillae are in tandem; in the remaining 2 species, the first 2 pairs of postcloacal papillae form a single line near posterior cloacal lip. In $P$. obtusissima, the third pair of sessile papillae is between the fourth pair of stalked papillae; in $P$. squamatae, the third pair of sessile papillae is posterior to the fourth pair of stalked papillae. We have assigned our specimens to $P$. retusa because the right spicule is strongly curved. With the exception of occurrences in the toads Rhinella granulosus and $R$. margaritifera reported by Goncalves et al. (2002), Physaloptera retusa is known only from lizards. Both Drymobius margaritiferus and Oxybelis fulgidus are known to feed on lizards (Savage, 2002). Because $P$. retusa is a stomach worm and was found in such low numbers $(1,1)$, and in the intestines of these snakes, it most likely is present due to diet. Drymobius margaritiferus and Oxybelis fulgidus represent new host records for $P$. retusa.

\section{Diplotriaenoidea \\ Diplotriaenidae (Skrjabin, 1916) Anderson, 1958 \\ Dicheilonematinae Wehr, 1935 Hastospiculum onchocercum Chitwood, 1932}

Hosts and locality: Boa constrictor (1): male, UT 02-P-0746, collected 16 August 2002, Area Administrative, Guanacaste Conservation Area.

Prevalence and intensity: 1 of 4 B. constrictor $(25 \%, 1)$.

Site of infection: Surface of stomach.

Additional Costa Rican records: Dendrophidion pericarinatus (Goldberg and Bursey, 2004a); Dendrophidion vinitor (Goldberg and Bursey, 2004a).

Type host and locality: Boa constrictor, zoo (Chitwood, 1932). 
Other reports: Boa constrictor (Caballero, 1947; Chitwood, 1932); Crotalus durissus (Desportes, 1941; Caballero, 1947; Araujo, 1970; Vicente et al., 1993); Epicrates cenchria (Everard, 1975); Philodryas aestivus (Vicente et al., 1993); Spilotes pullatus (Vicente and Jardim, 1980).

Geographic range: Brazil (Desportes, 1941); Costa Rica (Goldberg and Bursey, 2004a); Mexico (Caballero, 1947); Trinidad (Everard, 1975).

Specimen deposited: Boa constrictor, USNPC 103638; (vial).

\section{Remarks}

This is the third report of $H$. onchocercum in $\mathrm{Boa}$ constrictor.

\section{Filarioidea \\ Onchocercidae (Leiper, 1911) \\ Onchocercinae Leiper, 1911 \\ Macdonaldius oschei Chabaud and Frank, 1961}

Hosts and locality: Boa constrictor (1): UT 02-P0685, collected 16 August 2002, Interamerican Highway between Santa Rosa and Poco, Guanacaste Conservation Area.

Prevalence and intensity: 1 of 4 B. constrictor $(25 \%, 18)$.

Site of infection: Mesentery.

\section{Additional Costa Rican records: None.}

Type host and locality: Python molurus, Stuttgard Zoological Garden, Germany (Chabaud and Frank, 1961a).

Other reports: Boa constrictor (Frank, 1964a, b, c; Telford, 1965).

Geographic range: Costa Rica (this study); Mexico (Telford, 1965).

Specimen deposited: Boa constrictor, USNPC 103639; (vial).

\section{Remarks}

Macdonaldius oschei was described by Chabaud and Frank (1961a) from specimens taken from the major arteries of Python molurus bivittauts from India and Python reticulatus from Indonesia that had died in the Zoological Garden in Stuttgart, Germany. It was subsequently found in Boa constrictor in the same zoo and microfilariae were described (Chabaud and Frank, 1961b). Frank (1962, 1964a, b) reported that microfilariae invaded and developed in the Malphighian tubules of the New World argasid tick Ornithodoros talaju, which had been introduced to the zoo. Frank (1964c) concluded that $M$. oschei was normally a parasite of New World reptiles, and its appearance in other hosts in the Stuttgard Zoological Garden was the result of the introduction of suitable vectors along with infected reptiles. To our knowledge, Telford (1965) is the only previous report of wild-caught snakes harboring $M$. oschei. Costa Rica is a new locality record.

\section{STRONGYLIDA Diaphanocephaloidea Diaphanocephalidae Travassos 1920 Kalicephalus costatus (Rudolphi, 1819) Yorke and Maplestone, 1926 K. costatus costatus (Rudolphi, 1819)}

(Syn. Strongylus costatus Rudolphi, 1819; Kalicephalus strumosus Molin, 1861; Kalicephalus mucronatus Molin, 1861; Scelerostomum kalicephalium Stossich, 1899; Kalicephalus philodryadus Ortlepp, 1923.)

Hosts and locality: Imantodes cenchoa (1): female, UT 03-P-0263, collected 24 March 2003, Rio Negro, Guanacaste Conservation Area; Leptophis depressirostris (2): male, UT 01-P-0322, collected 22 June 2001 Rio Negro, Guanacaste Conservation Area; female, UT 03-P-0193, collected 24 February 2003, Rio Cucaracho, Guanacaste Conservation Area.

Prevalence and intensity: 1 of 1 I. cenchoa (100\%, 1); 2 of 4 L. depressirostris (50\%, 1, 6, respectively).

Site of infection: Large intestine.

Additional Costa Rican records: Boa constrictor (Schad, 1962).

Type host and locality: Coluber sp., Brazil (Rudolphi, 1819). No species of Coluber is currently listed for Brazil.

Other reports: Antillophis andreae (Barus and Coy Otero, 1978); Bothrops alternatus (Fernandes and Artigas, 1978); Bothrops atrox (Schad, 1962; DiazUngria, 1964); Bothrops cotiara (Fernandes and Artigas, 1978); Bothrops jararaca (Schad, 1962; Fernandes and Artigas, 1978); Bothrops jararacussu (Fernandes and Artigas, 1978); Bothrops pradoi (Fernandes and Artigas, 1978); Chironius carinatus 
Table 1. Costa Rican snake nematodes by reference: 1. this paper; 2. Viquez, 1935; 3. Brenes and Bravo-Hollis, 1960; 4. Schad, 1962; 5. Goldberg and Bursey, 2004a; 6. Goldberg and Bursey, 2006a.

\begin{tabular}{|c|c|c|c|c|c|c|c|c|}
\hline & \multicolumn{8}{|c|}{ Nematode } \\
\hline & $\begin{array}{l}\text { Abbreviata } \\
\text { costaricae }\end{array}$ & $\begin{array}{l}\text { Aplectana } \\
\text { incerta }\end{array}$ & $\begin{array}{l}\text { Aplectana } \\
\text { itzocanensis }\end{array}$ & $\begin{array}{l}\text { Cosmo- } \\
\text { cercoides } \\
\text { variabilis }\end{array}$ & $\begin{array}{l}\text { Cruzia } \\
\text { rudolphi }\end{array}$ & $\begin{array}{c}\text { Hastospi- } \\
\text { culum } \\
\text { onchocercum }\end{array}$ & $\begin{array}{c}\text { Hexametra } \\
\text { boddaertii }\end{array}$ & $\begin{array}{c}\text { Kalicephalus } \\
\text { costatus }\end{array}$ \\
\hline \multicolumn{9}{|l|}{ Boidae: } \\
\hline Boa constrictor & - & - & - & - & - & 1 & - & 4 \\
\hline \multicolumn{9}{|l|}{ Colubridae: } \\
\hline Chironius grandisquamis & - & 1 & - & - & - & - & - & - \\
\hline Conophis lineatus & - & 1 & - & - & - & - & - & - \\
\hline Dendrophidion percarinatus & - & - & - & 1 & - & 5 & - & - \\
\hline Dendrophidion vinitor & - & - & - & - & - & 5 & - & - \\
\hline Drymobius margaritiferus & - & - & 1 & - & - & - & - & - \\
\hline Erythrolamprus mimus & - & - & - & - & - & - & - & - \\
\hline Imantodes cenchoa & - & - & - & - & - & - & - & 1 \\
\hline Imantodes inornatus & - & - & - & - & - & - & - & - \\
\hline Leptodeira annulata & - & - & - & - & - & - & - & - \\
\hline Leptodrymus pulcherrimus & - & - & - & - & - & - & - & - \\
\hline Leptophis depressirostris & - & - & - & - & - & - & - & 1 \\
\hline Liophis epinephelus & - & - & 1 & - & - & - & - & - \\
\hline Masticophis mentovarius & 1 & - & - & - & - & - & - & - \\
\hline Nina maculata & - & - & - & 6 & - & - & - & - \\
\hline Oxybelis aeneus & - & - & - & - & - & - & - & - \\
\hline Oxybelis brevirostris & - & - & - & - & - & - & - & - \\
\hline Oxybelis fulgidus & - & - & - & - & - & - & - & - \\
\hline Pliocercus euryzonus & - & - & - & - & - & - & - & - \\
\hline Sibon nebulatus & - & - & - & - & 1 & - & - & - \\
\hline Spilotes pullatus & - & - & - & - & - & - & 1 & - \\
\hline Trimorphodon biscutatus & - & - & - & - & - & - & 1 & - \\
\hline Urotheca guentheri & - & - & - & - & - & - & - & - \\
\hline Xenodon rabdocepalus & - & - & - & - & - & - & - & - \\
\hline \multicolumn{9}{|l|}{ Elapidae: } \\
\hline Micrurus nigrocinctus & - & - & - & - & - & - & 5 & - \\
\hline \multicolumn{9}{|l|}{ Viperidae: } \\
\hline Agkistrodon bilineatus & 1 & - & - & - & - & - & 1 & - \\
\hline Atropoides nummifer & - & - & - & - & - & - & - & - \\
\hline Bothrops atrox & - & - & - & - & - & - & - & - \\
\hline Bothrops asper & - & - & - & - & - & - & - & - \\
\hline Crotalus durissus & - & - & - & 1 & - & - & 1 & - \\
\hline Porthidium nasutum & - & 1 & - & - & - & - & - & - \\
\hline Porthidium ophryomegas & - & 1 & - & - & - & - & 1 & - \\
\hline
\end{tabular}

(Schad, 1962); Chironius sexcarinatus (Schad, 1962); Crotalus durissus (Schad, 1962; Fernandes and Artigas, 1978); Cromicus angulifer (Schad, 1962); Erythrolampris venustimmimus (Schad, 1962); Eudryas bifossatus (Schad, 1962); Lachesis muta (Schad, 1962); Liophis miliaris (Schad, 1962; Fernandes and Artigas, 1978); Lystrophis semicinctus (Schad, 1962); Mastigodryas bifossatus (Fabio and Rolas, 1974); Philodryas patagoniensis (Fernandes and Artigas, 1978); Philodryas schotti (Schad, 1962); Philodryas serra (Schad, 1962); Phrynosoma sulphuraceus
(Schad, 1962); Thalerophis ahaetulla (Schad, 1962); Thalerophis occidentalis (Schad, 1962); Thalerophis richardi occidntalis (Schad, 1964); Waglerophis merremii (Fernandes and Artigas, 1978); Xenodon guentheri (Fernandes and Artigas, 1978); Xenodon neuwiedii (Fernandes and Artigas, 1978).

Geographic range: Brazil (Schad, 1962); British Guiana (Schad, 1962); Costa Rica (Schad, 1962); Cuba (Barus and Coy Otero, 1978); Ecuador (Schad, 1964). 
Table 1. Extended.

\begin{tabular}{|c|c|c|c|c|c|c|c|c|c|}
\hline \multicolumn{10}{|c|}{ Nematode } \\
\hline $\begin{array}{l}\text { Kalice- } \\
\text { phalus } \\
\text { inermis }\end{array}$ & $\begin{array}{l}\text { Kalice- } \\
\text { phalus } \\
\text { sublatus }\end{array}$ & $\begin{array}{l}\text { Macdo- } \\
\text { naldius } \\
\text { oscheri }\end{array}$ & $\begin{array}{l}\text { Ophida- } \\
\text { scaris } \\
\text { arndti }\end{array}$ & $\begin{array}{l}\text { Ophida- } \\
\text { scaris } \\
\text { sicki }\end{array}$ & $\begin{array}{c}\text { Physa- } \\
\text { loptera } \\
\text { obtusissima }\end{array}$ & $\begin{array}{l}\text { Physa- } \\
\text { loptera } \\
\text { retusa }\end{array}$ & $\begin{array}{l}\text { Skrjabine- } \\
\text { lazia } \\
\text { intermedia }\end{array}$ & $\begin{array}{l}\text { Terranova } \\
\text { caballeroi }\end{array}$ & $\begin{array}{c}\text { Travasso- } \\
\text { sascaris } \\
\text { araujoi }\end{array}$ \\
\hline - & 1,4 & 1 & - & - & - & - & - & - & - \\
\hline - & - & - & - & - & - & - & - & - & - \\
\hline- & - & - & - & - & - & - & - & - & - \\
\hline - & - & - & - & - & - & - & - & - & - \\
\hline - & - & - & - & - & - & - & - & - & - \\
\hline- & - & - & - & - & - & 1 & - & - & - \\
\hline- & - & - & - & - & - & - & - & 1 & - \\
\hline - & - & - & - & - & - & - & - & - & - \\
\hline - & - & - & - & - & - & - & - & - & - \\
\hline - & - & - & - & - & - & - & - & - & - \\
\hline - & - & - & - & - & - & - & - & - & - \\
\hline 1 & - & - & - & - & - & - & - & - & - \\
\hline - & - & - & - & - & - & - & - & 5 & - \\
\hline- & - & - & - & - & - & - & - & - & 1 \\
\hline - & - & - & - & - & - & - & - & - & - \\
\hline - & - & - & - & - & - & - & - & - & - \\
\hline- & - & - & - & - & - & - & - & - & - \\
\hline - & - & - & - & - & - & 1 & 1 & - & - \\
\hline- & - & - & - & - & - & - & - & - & - \\
\hline- & - & - & - & - & - & - & - & - & - \\
\hline- & - & - & - & - & - & - & - & - & - \\
\hline- & - & - & - & - & - & - & - & - & - \\
\hline 1 & - & - & - & - & - & - & - & - & - \\
\hline- & - & - & - & 1 & - & - & - & - & 1 \\
\hline- & - & - & - & - & - & - & - & - & - \\
\hline- & - & - & - & - & - & - & - & - & - \\
\hline 1 & - & - & - & - & - & - & - & - & - \\
\hline 3 & - & - & - & - & 2 & - & - & - & - \\
\hline- & - & - & - & - & - & - & - & - & - \\
\hline- & - & - & 1 & - & - & - & - & - & - \\
\hline 1 & - & - & - & - & - & - & - & - & - \\
\hline- & - & - & - & - & - & - & - & - & - \\
\hline
\end{tabular}

Specimen deposited: Imantodes cenchoa USNPC 103640; Leptophis depressirostris, USNPC 103641, 103642; (vials).

\section{Remarks}

Schad (1962) divided $K$. costatus into 4 subspecies based upon locality: $K$. costatus costatus, South and Central America; K. costatus parvus, North America; $K$ costatus micrurus, Tropical and South Africa; $K$. costatus indicus, Orient and Australasia. We have only listed hosts for $K$. costatus costatus. Imantodes cenchoa and Leptophis depressirostris represent new host records for $K$. costatus.

\section{Kalicephalus inermis Molin, 1861}

\section{K. inermis macrovulvus Caballero, 1954}

(Syn. Kalicephallus macrovulvus Caballero, 1954).

Hosts and locality: Atropoides nummifer (1): female, UT 04-P-0590, collected 21 November 2004, Estación, Guanacaste Conservation Area; Leptophis depressirostris (1): UT 05-P-0304 col- 
lected 26 September 2005, Brasilia, Guanacaste Conservation Area; Porthidium nasutum (1): male, UT 03-P-0400, collected 25 May 2003, San Gerardo, Guanacaste Conservation Area; Urotheca guentheri (1): female, UT 03-P-0290, collected 9 April 2003, Volcan Santa Maria, Guanacaste Conservation Area.

Prevalence and intensity: 1 of 1 A. nummifer $(100 \%$, 5); 1 of 4 L. depressirostris $(25 \%, 1) ; 1$ of $1 P$.nasutum (100\%, 1); 1 of 1 U. guentheri $(100 \%, 3)$.

Site of infection: Large intestine.

Additional Costa Rican records: Bothrops atrox (Brenes and Bravo-Hollis, 1960).

Type host and locality: Agkistrodon bilineatus, Guatemala (Caballero, 1954).

Other reports: Chironius multiventris (McAllister et al., 2010); Lachesis muta (Schad, 1962); Thalerophis occidentalis (Schad, 1962).

Geographic range: Costa Rica (Brenes and BravoHollis, 1960); Ecuador (McAllister et al., 2010); Guatemala (Caballero, 1954); Trinidad (Schad, 1962).

Specimen deposited: Atropoides nummifer, USNPC 103643; Leptophis depressirostris USNPC 103644; Porthidium nasutum, USNPC 103645; Urotheca guentheri, USNPC 103646; (vials).

\section{Remarks}

Schad (1962) erected an "inermis group" by reducing Kalicephalus inermis Molin, 1861, Kalicephalus macrovulvus Caballero, 1954, and Kalicephalus coronellae Ortlepp, 1923 to subspecies (geographic race) status: $K$. inermis inermis in Brazil; $K$. inermis macrovulvus in northern South America and Central America; and $K$. inermis coronellae in Mexico and North America. Fernandes and Artigas (1978) considered these subspecies to be valid species, but Baker (1987) retained the subspecies designation. Atropoides nummifer, Leptophis depressirostris, Porthidium nasutum, and Urotheca guentheri represent new host records for Kalicephalus inermis.

\section{Kalicephalus subulatus Molin, 1861}

(Syn. Ankylostoma boae Blanchard, 1886; Strongylus boae MacCallum, 1921; Kalicepahlus chitwoodi Caballero, 1954).

Hosts and locality: Boa constrictor (2): UT 02-P0685 collected 16 August 2002, Interamerican Highway, Guanacaste Conservation Area; female,
UT 05-P-0011, collected 25 May 2005, Camino a Naranjo, Guanacaste Conservation Area.

Prevalence and intensity: 2 of 4 B. constrictor (50\%, 19, 2, respectively).

Site of infection: Large intestine.

Additional Costa Rican records: Boa constrictor (Schad, 1962; Rodriguez-Ortiz et al., 2003).

Type host and locality: Boa constrictor, Brazil (Molin, 1861).

Other reports: Boa constrictor (Caballero and Vogelsand, 1950; Caballero, 1954; Ubelaker and Dailey, 1966; Everard, 1975; Fernandes and Artigas, 1975); Corallus caninus (Fernandes and Artigas, 1975); Epicrates cenchria (Fernandes and Artigas, 1975); Lachesis muta (Goncalves et al., 2002).

Geographic range: Brazil (Molin, 1861); Costa Rica (Schad, 1962) Guatemala (Caballero, 1954); Trinidad (Everard, 1975); Venezuela (Caballero and Vogelsand, 1950).

Specimen deposited: Boa constrictor, USNPC 103647, 103648; (vials).

\section{Remarks}

In his monograph, Schad (1962) restricted the name Kalicephalus subulatus to species occurring in the Boa constrictor. More recently, Fernandes and Artgas (1975) and Goncalves et al. (2002) have determined additional hosts. This is the third report of $K$. subulatus from Costa Rica.

\section{DISCUSSION}

Of the 133 snake species found in Costa Rica (Savage, 2002), 40 (30\%) have so far been found to harbor nematodes. One additional record of nematodes in Costa Rican snakes should be noted: Goldberg and Bursey (2004a) reported larvae of Contracaecum, a bird parasite, in Erytrolampus bizona and Micurus alleni. Table 1 lists the currently known species of nematodes from Costa Rican snakes; we have not included undetermined species or larval forms in the table. The current information is too sparse to draw general conclusions, but it would appear that 3 categories of nematodes are present: those that parasitize snakes sensu stricto, namely Abbreviata costaricae, Cruzia rudolphi, Hastospiculum onchocercum, Hexametra boddaertii, Kalicephalus costatus, K. inermis, K. sublatus, Macdonaldius 
oscheri, Ophidascaris arndti, O. sicki, Physaloptera obtusissima, Terranova caballeroi, Travassosascaris araujoi; those present as a by-product of diet and not parasites sensu stricto (we are hesitant to use the terms "accidental" or "incidental" because we were unable to determine if these nematodes were alive within the intestine of the host), Aplectana incerta, Aplectana itzocanensis, Cosmocercoides variabilis Physaloptera retusa, Skrjabinelazia intermedia; and those using snakes as paratenic hosts, Contracaecum, Porrocaecum. Further examination of Costa Rican snakes will be necessary before categorization of the helminth community can be made.

\section{ACKNOWLEDGMENTS}

We thank the scientific and technical staff of the Area de Conservación Guanacaste (ACG) for support of this study, in particular: Elda Araya, Roger Blanco, Duvalier Briceño, Carolina Cano, Maria Marta Chavarría, Felipe Chavarría, Roberto Espinoza, Dunia Garcia, Guillermo Jimenez, Elba Lopez, Sigifredo Marin, Alejandro Masis, Calixto Moraga, Fredy Quesada, and Petrona Rios. Thanks also to Dan Janzen and Winnie Hallwachs, scientific advisers to the ACG, for their support. This study was funded by a Discovery Grant from the Natural Sciences and Engineering Research Council (NSERC) of Canada to D.R.B.

\section{LITERATURE CITED}

Anderson, R. M. 2000. Nematode Parasites of Vertebrates: Their Development and Transmission, 2nd ed. CABI Publishing, Wallingford, Oxon, U.K. 650 pp.

Andrews, K. D., R. L. Lampley, M. A. Gillman, D. T. Corey, S. R. Ballard, M. J. Blasczyk, and W. G. Dyer. 1992. Helminths of Rana catesbeiana in southern Illinois with a checklist of helminths in bullfrogs of North America. Transactions of the Illinois State Academy of Science 85:147-172.

Araujo, P. 1969. Nouvelle espece d'Ophidascaris Baylis 1921, parasite du serpent crotale (Crotalus durissus terrificus). Annales de Parasitologie (Paris) 44:441-449.

Araujo, P. 1970. Considerações sobre Hastospiculum onchocercum major Desportes, 1941, parasita da serpente Crotalus durissus terrificus. Revista de Farmacia e Bioqueimica da Universidade de Sao Paulo 8: 121-124.

Avila, R. W., and R. J. da Silva. 2009. Helminths of the teiid lizard Kentropyx calcarata (Squamata) from an Amazonian site in western Brazil. Journal of Helminthology 83:267-269.

Avila, R. W., L. A. dos Anjos, R. J. Silva, H. C. Costa, V. Pedro, and R. N. Feio. 2010. Tupinambis longilineus (NCN). Endoparasites. Herpetological Review 41:87-88.

Avila, R. W., F. L. Souza, and R. J. da Silva. 2010. Helminths from seven species of lizards (Reptilia:
Squamata) at the Cerrado of Mato Grosso do Sul State, Brazil. Comparative Parasitology 77:67-71.

Baird, W. 1860. Description of some new species of intestinal worms (Entozoa) in the collection of the British Museum. Proceedings of the Zoological Society, London 28:446-448.

Baker, M. R. 1985. Redescription of Aplectana itzocanensis and $A$. incerta (Nematoda: Cosmocercidae) from Amphibians. Transactions American Microscopical Society 104:272-277.

Baker, M. R. 1987. Synopsis of the Nematoda parasitic in amphibians and reptiles. Memorial University of Newfoundland, Occasional Papers in Biology 11:1325 .

Barus, V. 1973. Nematodes parasitizing hosts of the genus Bufo (Amphibia) in Cuba. Folia Parasitologica (PRAHA) 20:29-39.

Barus, V., and A. Coy Otero. 1966. Nota sobre la helminthofauna de ofidios en Cuba: Descripciones de tres especies nuevas de nematodos. Poeyana 23:1-16.

Barus, V., and A. Coy Otero. 1968. Freitasia leixeirai gen. n. et sp.n. and other nematodes parasitizing Anolis equestrus (Squamata: Iguanidae). Folia Parasitologica (Praha) 15:41-45.

Barus, V., and A. Coy Otero. 1969. Systematic survey of nematodes parasitizing lizards (Sauria) in Cuba. Helminthologia 10:329-346.

Barus, V., and A. Coy Otero. 1978. Nematodes parasitizing Cuban snakes (Ophidia). Vestnik Ceskoslovenske Spolecnosti Zoologicke 42:85-100.

Bolek, M. G., and J. R. Coggins. 1998. Endoparasites of Cope's gray treefrog, Hyla chrysoscelis, and western chorus frog, Pseudacris $t$. triseriata, from southeastern Wisconsin. Journal of the Helminthological Society of Washington 65:212-218.

Bolek, M. G., and J. R. Coggins. 2000. Seasonal occurrence and community structure of helminth parasites from the eastern American toad, Bufo americanus americanus, from southeastern Wisconsin, U.S.A. Comparative Parasitology 67:202-209.

Bowman, D. D. 1984. Hexametra leidyi sp. n. (Nematoda: Ascarididae) from North American pit vipers (Reptilia: Viperidae). Proceedings of the Helminthological Society of Washington 51:54-61.

Brandt, B. B. 1936. Parasites of certain North Carolina Salientia. Ecological Monographs 6:492-532.

Bravo-Hollis, M. 1943. Dos nuevos nematodos parasitos de anuros del sur de Puebla. Anales del Instituto de Biologia, Universidad Nacional Autonoma de Mexico, Serie Zoologia 14:69-78.

Brenes, R. R., and M. Bravo-Hollis. 1959. Helmintos de la Republica de Costa Rica VIII. Nematoda 2. Algunos nematodes de Bufo marinus marinus (L) y algunas considseraciones sobre los generos Oxysomatium y Aplectana. Review Tropical Biology 7:35-55.

Brenes, R. R., and M. Bravo-Hollis. 1960. Helmintos de la Republica de Costa Rica IX. Nematoda 3. Algunos nematodos de reptiles, con descripcion de dos nuevas especies: Atractis caballeroi n. sp. y Cyrtosomum longicaudatum n. sp. Libro Homenaje al Dr. Eduardo Caballero y Caballero. Instituto Politechnico Nacional, Esquela Nacional de Ciencias Biologicas, Mexico. pp. 451-464. 
Bursey, C. R., and D. R. Brooks. 2010a. Nematode parasites of 41 anuran species from the Area de Conservación Guanacaste, Costa Rica. Comparative Parasitology 77:229-239.

Bursey, C. R., and D. R. Brooks. 2010b. Nematode parasites of 16 lizard species from the Area de Conservación Guanacaste, Costa Rica. Comparative Parasitology 77:240-243.

Bursey, C. R., and W. F. DeWolf II. 1998. Helminths of the frogs Rana catesbiana, Rana clamitans, and Rana palustris from Coshocton County, Ohio. Ohio Journal of Science 98:28-29.

Bursey, C. R., and S. R. Goldberg. 1991. Monthly prevalences of Physaloptera retusa in naturally infected Yarrow's spiny lizard. Journal of Wildlife Diseases 27:710-715.

Bursey, C. R., and S. R. Goldberg. 1994. Persistence of the component parasite community of Yarrow's spiny lizard, Sceloporus jarrovii, 1967-1991. Journal of the Helminthological Society of Washington 61:141-145.

Bursey, C. R., and S. R. Goldberg. 1998. Helminths of the Canadian toad, Bufo hemiophrys (Amphibia: Anura), from Alberta, Canada. Journal of Parasitology 84:617618.

Bursey, C. R., and S. R. Goldberg. 2004. Helminths of Tropidurus guarani (Sauria: Tropiduridae) from Paraguay. Comparative Parasitology 71:203-207.

Bursey, C. R., and S. R. Goldberg. 2005. New species of Oswaldocruzia (Nematoda: Molineoidae), new species of Rhabdias (Nematoda: Rhabdiasidae) and other helminths in Rana cf. forreri (Anura: Ranidae) from Costa Rica. Journal of Parastiology 91:600-605.

Bursey, C. R., and S. R. Goldberg. 2006a. New species of Raillietnema (Nematoda: Cosmocercidae) and other helminths in Rana vibicaria (Ranidae) from Costa Rica. Comparative Parasitology 73:193-200.

Bursey, C. R., and S. R. Goldberg. 2006b. Helminths in Mesaspis monticola (Squamata: Anguidae) from Costa Rica, with the description of a new species of Entomelas (Nematoda: Rhabdiasidae) and a new species of Skrjabinodon (Nematoda: Pharyngodonidae). Parasite 13:183-191.

Bursey, C. R., and S. R. Goldberg. 2007. New species of Hedruris (Nematoda: Hedruridae), Anuracanthorhynchus lutzi (Hamann, 1891) n. comb. and other helminths in Lithobates warszewitschii (Anura: Ranidae) from Costa Rica. Caribbean Journal of Science 43: $1-10$.

Bursey, C. R., S. R. Goldberg, and J. B. Bettaso. 2010. Persistence and stability of the component helminth community of the foothill yellow-legged frog, Rana boylii (Ranidae), from Humboldt County, California, 1964-1965 versus 2004-2007. American Midland Naturalist 163:476-482.

Bursey, C. R., S. R. Goldberg, and J. R. Parmelee. 2001. Gastrointestinal helminths of 51 species of anurans from Reserva Cuzco Amazónico, Peru. Comparative Parasitology 68:21-35.

Bursey, C. R., S. R. Goldberg, and J. R. Parmelee. 2005. Gastrointestinal helminths from 13 species of lizards from Reserva Cuzco Amazónico, Peru. Comparative Parasitology 72:50-68.

Bursey, C. R., S. R. Goldberg, and S. M. Secor. 1995. Hexametra boddaertii (Nematoda: Ascaridae) in the sidewinder, Crotalus cerastes (Crotalidaae), from California. Journal of the Helminthological Society of Washington 62:78-80.

Bursey, C. R., S. R. Goldberg, and S. R. Telford, Jr. 2007. Gastrointestinal helminths of 14 species of lizards from Panama with descriptions of five new species. Comparative Parasitology 74:108-140.

Bursey, C. R., and D. R. Schibli. 1995. A comparison of the helminth fauna of two Plethodon cinereus populations. Journal of the Helminthological Society of Washington 62:232-236.

Caballero, E. 1947. Algunas filarias de mamiferos y de reptiles de las republicas de Colombia y Panama. Anales de Instsituto de Biologia de la Universidad Nacional de Mexico 18:169-188.

Caballero, E. 1949. Estudios helmintologicos de la region Oncocercosa de Mexico y de la Republic de Guatemala. Nematoda, 5th parte. Anales de Instituto de Biologica 20:279-292.

Caballero, E. 1951. Estudios helmintologicos de la region Oncocercosa de Mexico y de la Republica de Guatemala. Nematoda. 6a parte. Y alganas consideraciones en torno a los generos Onchocerca Diesing, 1841. Y Acanthospicullum Skrjabin y Schikhobalowa, 1948. Anales de Instituto de Biologia de la Universidad Nacional de Mexico 22:141-158.

Caballero, E. 1954. Estudios helmintologicos de la region Oncocercosa de Mexico y de la Republica de Guatemala. Nematoda. 8th Parte. Anales del Instituto de Biologia de la Universidad Nacional Autonoma de Mexico. 25:259-274.

Caballero, E., and E. G. Vogelsang. 1947. Fauna Helmintologica venezolana. I. Ochetosoma miladelarocai n. sp., de Bothrps atrox L. y hallazgo de Physaloptera retusa (Rud., 1819) en Cnemidophorus lemniscatus lemniscatus. Revista de medicina veterinaria y parasitologia 6:53-62.

Caballero, E., and E. G. Vogelsang. 1950. Fauna helmintologica venezolana. III. Algunos nematodos de animales silvestres. Revista de medicina veterinaria y parasitologia 9:55-67.

Caballero Deloya, J. 1974. Estudio helmintologico de los animales silvestres de la Estacion de Bioloia Tropical "Los Tuxtlas" Veracruz. Nematoda I. Algunos nematododos parasitos de Bufo horribilis Wiegmann, 1833. Anales del Instituto de Biologic de la Universidad Nacional Autonmoma de Mexico 43:45-50.

Campbell, R. A. 1968. A comparative study of the parasites of certain Salientia from Pocahontas State Park, Virginia. Virginia Journal of Science 19:13-20.

Chabaud, A. G., and M. Frank. 1961a. Nouvelle filaire parasite des artères de pythons; Macdonaldius oschei $\mathrm{n}$. sp. (Nématodes, Onchocercidae. Zeitschrift für Parasitenkunde 20:434-439.

Chabaud, A. G., and M. Frank. 1961b. Description de la microfilaire de Macdonaldius oschei. Annales de Parasitologie Humaine et Comparee 36:133-134.

Chitwood, B. G. 1932. A review of the nematodes of the genus Hastospiculum, with descriptions of two new species. Proceedings of the U. S. National Museum 80:1-9.

Coggins, J. R., and R. A. Sajdak. 1982. A survey of helminth parasites in the salamanders and certain anurans from Wisconsin. Proceedings of the Helminthological Society of Washington 49:99-102. 
Coy Otero, A. 1970. Contribucion al conocimiento de la helmintofauna de los saurios cubanos. Ciencias 4:1-58.

Coy Otero, A., and U. Barus. 1979. Nematodes parasitizing Cuban reptiles. Acta Scientiarum Naturalium Academiae Scientiarum Bohemoslovacae Brno 13:3-43.

Coy Otero, A., and L. Ventosa. 1984. Nematodos parasitos de anfibios cubanos. Poeyana 269:1-20.

Criscione, D. D., and W. F. Font. 2001. The guest playing host: colonization of the introduced Mediterranean gecko, Hemidactylus turcicus, by helminth parasites in southeastern Louisiana. Journal of Parasitology 87: $1273-1278$.

Cristofaro, R., J. F. Guimaraes, and H. Oliveira Rodrigues. 1976. Alguns nematodeos de Tropidurus torquatus (Wied) e Ameiva ameiva (L.) -Fauna Helmintologica de Salvador, Bahia. Atas Sociedade de Biologia de Rio de Janeiro 18:65-70.

Desportes, C. 1941. Nouvelles recherches sur la morphologie et sur l'evolution d'Icosiella neglecta (Diesing 1851), filaire commune de la grenouille verte. Annales de Parasitologie humaine et comparee 18:46-66.

Dias, E. J. R., D. Vrcibradic, and C. F. D. Rocha. 2005. Endoparasites infecting two species of whiptail lizard (Cnemidophorus abaetensis and C. ocellifer; Teiidae) in a 'restinga' habitat of northeastern Brazil. Herpetological Journal 15:133-137.

Diaz-Ungria, C. 1964. Notas sobre nematodes de reptiles en Venezuela. Revesta Veterenaria Venezolana 17:402.

Diaz-Ungria, C., and M. F. Gallardo. 1968. Hematodes de reptiles Venezolanos, con descripcion de varias especies nuevas. Boletain Sociedad Venezolana de Ciencias Naturales 27:550-570.

Espínola-Novelo, J. F., and S. Guillén-Hernández. 2008. Helminth parasites in Chaunus marinus and Cranopsis valliceps (Anura: Bufonidae) from Lagunas Yalahau, Yucatan, Mexico. Journal of Parasitology 94:672674.

Espinoza-Jiménez, A., L. Garcéa-Prieto, D. OsorioSarabia, and V. León-Règagnon. 2007. Checklist of helminth parasites of the cane toad Bufo marinus (Anura: Bufonidae) from Mexio. Journal of Parasitology 93:937-944.

Everard, C. O. R. 1975. Endoparasitaes of some Amphibia, reptiles and small mammals from Trinidad. Journal of the Trinidad and Tabago Field Naturalists' Club. pp. 72-79.

Fabio, S. P., and F. J. T. Rolas. 1974. Sobre alguns helmintos parasitos de Dryadophis bifossatus (Raddi). Memorias do Instituto Oswaldo Cruz 72:49-61.

Fernandes, M. P. M., and P. T. Artigas. 1978. Kalicepaluls inermis Molin, 1861 (Nematoda: Diaphanocephalidae) Rediscricao e confirmacao desta especie; informacoes de natureza biologica e critica do grupo 'inermis' proposto por Schad. Mem. Inst. Butanian 40/ 41:281-297.

Fontenot, L. W., and W. F. Font. 1996. Helminth parasites of four species of aquatic snakes from two habitats in southeastern Louisiana. Journal of the Helminthological Society of Washington 63:66-75.

Foster, G. W., P. E. Moler, J. M. Kinsella, S. P. Terrell, and D. J. Forester. 2000. Parasites of eastern indigo snakes (Drymarchon corais couperi) from Florida, U.S.A. Comparative Parasitology 67:124-128.
Frandsen, J. C., and A. W. Grundmann. 1960. The parasites of some amphibians of Utah. Journal of Parasitology 46:678.

Frank, W. 1962. Biologie von Macdonaldius oschei (Filarioidea, Onchocercinae), zugleich ein Beitrag über die wirtsspezifität von Ornithodoros talaje (Ixodoidea Argasidae). Zeitschrift für Parasitenkunde 22:107-108.

Frank, W. 1964a. Die entwicklung von Macdonaldius oschei Chabaud et Frank 1961 (Filarioidea, Onchocercidae) in der lederzecke Ornithodonros tajaje GuérinMéneville (Ixodoidea, Argasidae). Zeitschrift für Parasitenkunde 24:319-350.

Frank, W. 1964b. Die pathogenen wirkungen von Macdonaldius oscheri Chabaud et Frank 1961 (Filarioidea, Onchocercidae) bei verschiedened arten von Schlangen (Reptilia, Ophidia). Zeitschrift für Parasitenkunde 24: 249-275.

Frank, W. 1964c. Die Übertragung der Filarien-Infektionsstadien von Macdonaldius oschei Chabaud et Frank 1961 (Filarioidea, Onchocercidae) durch Ornitholoros talaje (Ixoidea, Argasidae) auf den Endwirt; Zugleich ein Beitrag zur Biologie des Übserträgers. Zeitschrift für Parasitenkunde 24:415-441.

Freitas, J. F. T. 1940. Sobre um interessante nematodeo parasito de reptil (Spiruroidea). Memorias do Instituto Oswaldo Cruz 35:603-605.

Freitas, J. F. T. 1951. Ophidascaris sicki n. sp. (Nematoda, Ascaroidea). Revista Brasileria de Biologie 11:255258.

Freitas, J. F. T. 1955. Redescricao de Ophidascaris ardti Sprehn, 1929 (Nematoda. Ascaroidea). Revista Iberica de Parsitologia Tomo Extra:17-22.

Galicia-Guerrero, S., C. R. Bursey, S. R. Goldberg, and G. Salgado-Maldonado. 2000. Helminths of two sympatric toad species, Bufo marinus (Linnaeus) and Bufo marmoreus Wiegmann, 1833 (Anura: Bufonidae) from Chamela, Jalisco, Mexico. Comparative Parasitology 67:129-133.

Goldberg, S. R., and C. R. Bursey. 1989a. Helminths of the giant spotted whiptail, Cnemidophorus burti stictogramus (Sauria: Teiidae). Proceedings of the Helminthological Society of Washington 56:86-87.

Goldberg, S. R., and C. R. Bursey. 1989b. Physaloptera retusa (Nematoda, Physalopteridae) in naturally infected sagebrush lizards, Sceloporus graciosus (Iguanidae). Journal of Wildlife Diseases 25:425-429.

Goldberg, S. R., and C. R. Bursey. 1990. Gastrointestinal helminths of the Yarrow spiny lizard, Sceloporus jarrovii jarrovii Cope. American Midland Naturalist 124:360-365.

Goldberg, S. R., and C. R. Bursey. 1991a. Helminths of three toads, Bufo alvarius, Bufo cognatus (Bufonidae), and Scaphiopus couchii (Pelobatidae), from southern Arizona. Journal of the Helminthological Society of Washington 58:142-146.

Goldberg, S. R., and C. R. Bursey. 1991b. Helminths of the red-spotted toad, Bufo punctatus (Anura: Bufonidae) from southern Arizona. Journal of the Helminthological Society of Washington 58:267-269.

Goldberg, S. R., and C. R. Bursey. 1991c. Gastrointestinal helminths of the northwestern alligator lizard, Gerrhonotus coeruleus principis (Anguidae). Journal of the Helminthological Society of Washington 58:246-248. 
Goldberg, S. R., and C. R. Bursey. 1996. Helminths of the oak toad (Bufo quercicus, Bufonidae) from Florida (U.S.A.). Alytes 14:122-126.

Goldberg, S. R., and C. R. Bursey. 2002a. Helminth parasites of seven anuran species from northwestern Mexico. Western North American Naturalist 62:160 169.

Goldberg, S. R., and C. R. Bursey. 2002b. Helminths of the plains spadefoot, Spea bombifrons, the western spadefoot, Spea hammondii, and the Great Basin spadefoot, Spea intermontana (Pelobatidae). Western North American Naturalist 62:491-495.

Goldberg, S. R., and C. R. Bursey. 2002c. Helminths of the bullfrog, Rana catesbeiana (Ranidae), in California with revisions to the California Anuran helminth list. Bulletin of the Southern California Academy of Science 101:18-130.

Goldberg, S. R., and C. R. Bursey. 2003. Helminths of two anuran species, Atelopus spurrelli (Bufonidae) and Dendrobates histrionicus (Dendrobatidae), from Colombia, South America. Parasitology International 52: 251-252.

Goldberg, S. R., and C. R. Bursey. 2004a. Coelomic metazoan endoparasites of 15 colubrid and two elapid snake species from Costa Rica. Caribbean Journal of Science 40:62-69.

Goldberg, S. R., and C. R. Bursey. 2004b. Norops pentaprion (Lichen anole). Endoparasites. Herpetological Review 5:269.

Goldberg, S. R., and C. R. Bursey. 2004c. Thamnophis validus (Mexican Pacific lowlands gartersnake). Endoparasites. Herpetological Review 35:75.

Goldberg, S. R., and C. R. Bursey. 2004d. Norops cupreus (Copper anole). Endoparasites. Herpetological Review 35:394.

Goldberg, S. R., and C. R. Bursey. 2005a. Coelomic helminths in the speckled racer, Drymobius margaritiferus (Serpentes: Colubridae) from Central America. Texas Journal of Science 57:103-106.

Goldberg, S. R., and C. R. Bursey. 2005b. Scaphiopus couchii (Couch's spadefoot). Endoparasites. Herpetological Review 36:165-166.

Goldberg, S. R., and C. R. Bursey. 2006a. Ninia maculata (Spotted coffee snake). Endoparasites. Herpetological Review 37:479-480.

Goldberg, S. R., and C. R. Bursey. 2006b. Mastigodryas melanolomus (Salmon-bellied racer). Endoparasites. Herpetological Review 37:478-479.

Goldberg, S. R., and C. R. Bursey. 2007a. Coelomic helminths of five colubrid snake species (Serpentes, Colubridae) from Costa Rica. Phyllomedusa 6:6972

Goldberg, S. R., and C. R. Bursey. 2007b. Helminths of two species of frogs, Lithobates taylori and Lithobates vaillanti (Ranidae), from Costa Rica. Caribbean Journal of Science 43:65-72.

Goldberg, S. R., and C. R. Bursey. 2007c. Uracentron flaviceps (Thornytail iguana). Endoparasites. Herpetological Review 38:85.

Goldberg, S. R., and C. R. Bursey. 2008. Helminths from 10 species of brachycephalid frogs (Anura: Brachycephalidae) from Costa Rica. Comparative Parasitology 75:255-262.
Goldberg, S. R., and C. R. Bursey. 2009a. Imantodes cenchoa (blunt-headed tree snake), Imanodoes gemmistratus (Central American tree snake), Imantodes inornatus (western tree snake). Endoparasites. Herpetological Review 40:230.

Goldberg, S. R., and C. R. Bursey. 2009b. Coelomic helminths in the northern cat-eyed snake, Leptodeira septentrionalis (Serpentes: Colubridae) from Costa Rica. Herpetological Bulletin 108:24-26.

Goldberg, S. R., and C. R. Bursey. 2009c. Helminths from seven species of Microlophus (Squamata: Tropiduridae) from Peru. Salamandra 45:125-128.

Goldberg, S. R., and C. R. Bursey. 2009d. Ameiva festiva (Middle American ameiva). Endoparasites. Herpetological Review 40:81-82.

Goldberg, S. R., and C. R. Bursey. 2010. Helminth biodiversity of Costa Rican anurans (Amphibia: Anura). Journal of Natural Histosry 44:1755-1787.

Goldberg, S. R., C. R. Bursey, and K. R. Beaman. 2004. Elgaria paucicarinata (San Lucan Alligator Lizard). Endoparasites. Herpetological Review 35:267.

Goldberg, S. R., C. R. Bursey, K. R. Beaman, and E. A. Dugan. 2006. Crotalus basiliscus (Mexican West Coast rattlesnake). Endoparasites. Herpetological Review 37:94

Goldberg, S. R., C. R. Bursey, and R. L. Bezy. 1996. Gastrointestinal helminths of Yarrow's spiny lizard, Sceloporus jarrovii (Phrynosomatidae) in Mexico. American Midland Naturalist 135:299-309.

Goldberg, S. R., C. R. Bursey, J. P. Caldwell, L. J. Vitt, and G. C. Costa. 2007. Gastrointestinal helminths from six species of frogs and three species of lizards sympatric in Para state, Brazil. Comparative Parasitology 74:327-342.

Goldberg, S. R., C. R. Bursey, and J. L. CamarilloRangel. 1999. Helminths of two lizards, Barisia imbricata and Gerrhonotus ophiurus (Sauria: Anguidae), from Mexico. Journal of the Helminthological Society of Washington 66:205-208.

Goldberg, S. R., C. R. Bursey, and J. L. CamarilloRangel. 2003. Gastrointestinal helminths of seven species of sceloporine lizards from Mexico. Southwestern Naturalist 48:208-217.

Goldberg, S. R., C. R. Bursey, and H. Cheam. 1995. Helminth parasites of three sympatric lizards from Grand Cayman Island, Anolis conspersus, Anolis sagrei (Polychridae) and Leiocepalus carinatus (Tropiduridae). Caribbean Journal of Science 31:339-340.

Goldberg, S. R., C. R. Bursey, and H. Cheam. 1997a. Helminths of 12 species of Anolis lizards (Polychrotidae) from the Lesser Antilles, West Indies. Journal of the Helminthological Society of Washington 64:248 257.

Goldberg, S. R., C. R. Bursey, and H. Cheam. 1997b. Persistence and stability of the component helminth community of the sagebrush lizard, Sceloporus graciosus (Phrynosomatidae) from Los Angeles County, California, 1972-1973, 1986-1996. American Midland Naturalist 138:418-421.

Goldberg, S. R., C. R. Bursey, and H. Cheam. 1998a Nematodes of the Great Plains narrow-mouthed toad, Gastrophryne olivacea (Microhylidae), from southern Arizona. Journal of the Helminthological Society of Washington 65:102-104. 
Goldberg, S. R., C. R. Bursey, and H. Cheam. 1998b. Composition and structure of helminth communities of the salamanders, Aneides lugubris, Batrachoseps nigriventris, Ensatina eschscholtzii (Plethodontidae), and Taricha torosa (Salamandridae) from California. Journal of Parasitology 84:248-251.

Goldberg, S. R., C. R. Bursey, and H. Cheam. 1998c. Helminths of the lizard Anolis cristatellus (Polychrotidae) from the British Virgin Islands, West Indies. Journal of the Helminthological Society of Washington 65:259-262.

Goldberg, S. R., C. R. Bursey, and H. Cheam. 1998d. Helminths of six species of Anolis lizards (Polychrotidae) from Hispaniola, West Indies. Journal of Parasitology 84:1291-1295.

Goldberg, S. R., C. R. Bursey, and H. Cheam. 1998e. Composition of helminth communities in montane and lowland populations of the western fence lizard, Sceloporus occidentalis from Los Angeles County, California. American Midland Naturalist 140:186-191.

Goldberg, S. R., C. R. Bursey, and H. Cheam. 1999. Helminths of the Madrean alligator lizard, Elgaria kingii (Sauria: Anguidae), from Arizona. Great Basin Naturalist 59:198-200.

Goldberg, S. R., C. R. Bursey, and M. D. Dailey. 2009. Taricha rivularis (Red-bellied newt). Endoparasites. Herpetological Review 40:331-332.

Goldberg, S. R., C. R. Bursey, and G. Galindo. 1999. Helminths of the lowland burrowing treefrog, Ptyernohyla fodiens (Hylidae), from Southern Arizona. Great Basin Naturalist 59:195-197.

Goldberg, S. R., C. R. Bursey, and E. W. A. Gergus. 2001. Helminth communities of subpopulations of the Pacific treefrog, Hyla regilla (Hylidae), from Baja California Mexico. Southwestern Naturalist 46:223230.

Goldberg, S. R., C. R. Bursey, and S. Hernandez. 1999. Helminths of the western toad, Bufo boreas (Bufonidae) from southern California. Bulletin of the Southern California Academy of Science 98:39-44.

Goldberg, S. R., C. R. Bursey, and A. T. Holycross. 2001. Sistrurus catenatus edwardsii (Desert massasauga). Endoparasites. Herpetological Review 32:265.

Goldberg, S. R., C. R. Bursey, and H. Kaiser. 1998. Gastrointestinal helminths of five species of Eleutherodactylus (Anura: Leptodactylidae) from the West Indies. Caribbean Journal of Science 34:146-149.

Goldberg, S. R., C. R. Bursey, K. B. Malmos, B. K. Sullivan, and H. Cheam. 1996. Helminths of the southwestern toad, Bufo microscaphus, Woodhouse's toad, Bufo woodhousii (Bufonidae), and their hybrids from central Arizona. Great Basin Naturalist 56:369374.

Goldberg, S. R., C. R. Bursey, and C. T. McAllister. 1995. Gastrointestinal helminths of nine species of Sceloporus lizards (Phrynosomatidae) from Texas. Journal of the Helminthological Society of Washington 62:188-196.

Goldberg, S. R., C. R. Bursey, R. G. McKinnell, and I. S. Tan. 2001. Helminths of northern leopard frogs Rana pipiens (Ranidae) from North Dakota and South Dakota. Western North American Naturalist 61:248251.
Goldberg, S. R., C. R. Bursey, and M. Morando. 2004. Metazoan endoparasites of 12 species of lizards from Argentina. Comparative Parasitology 71:208-210.

Goldberg, S. R., C. R. Bursey, and C. W. Painter. 2002. Helminths of the western diamondback rattlesnake, Crotalus atrox, from southeast New Mexico rattlesnake roundups. Southwestern Naturalist 47:307-310.

Goldberg, S. R., C. R. Bursey, and J. E. Platz. 2000. Helminths of the Plains leopard frog, Rana blairi (Ranidae). Southwestern Naturalist 45:362-366.

Goldberg, S. R., C. R. Bursey, and I. Ramos. 1995. The component parasite community of three sympatric toad species, Bufo cognatus, Bufo debilis (Bulfonidae), and Spea multiplicata (Pelobatidae) from New Mexico. Helminthological Society of Washington 62:57-61.

Goldberg, S. R., C. R. Bursey, G. Salgado-Maldonado, R. Baez, and C. Caneda. 2002. Helminth parasites of six species of anurans from Los Tuxtlas and Catemaco Lake, Veracruz, Mexico. Southwestern Naturalist 47: 293-299.

Goldberg, S. R., C. R. Bursey, B. K. Sullivan, and Q. H. Truong. 1996. Helminths of the Sonoran green toad, Bufo retiformis (Bufonidae), from southern Arizona. Journal of the Helminthological Society of Washington 63:120-122.

Goldberg, S. R., C. R. Bursey, and R. Tawil. 1993. Gastrointestinal helminths of the crevice spiny lizard, Sceloporus poinsetti (Phrynosomatidae). Journal of the Helminthological Society of Washington 60:263-265.

Goldberg, S. R., C. R. Bursey, and R. Tawil. 1994a. Helminth parasites of the bark anole, Anolis distichus and the brown anole, Anolis sagrei (Polychridae) from Florida and the Bahamas. Caribbean Journal of Science 30:275-277.

Goldberg, S. R., C. R. Bursey, and R. Tawil. 1994b. Gastrointestinal helminths of Sceloporus lizards (Phrynosomatidae) from Arizona. Journal of the Helminthological Society of Washington 61:73-83.

Goldberg, S. R., C. R. Bursey, and L. J. Vitt. 2006. Helminths of the brown-eared anole, Norops fuscoauratus (Squamata, Polychrotidae), from Brazil and Ecuador, South America. Phyllomedusa 5:83-86.

Goldberg, S. R., C. R. Bursey, and L. J. Vitt. 2009. Diet and parasite communities of two lizard species, Plica plica and Plica umbra from Brazil and Ecuador. Herpetological Journal 19:49-52.

Goldberg, S. R., J. Reyes-Velasco, C. R. Bursey, and E. Dugan. 2008. Porthidium hespere (Western hognose viper). Endoparasites. Herpetological Review 39:99.

Goncalves, A. Q., J. J. Vicente, and R. M. Pinto. 2002. Nematodes of Amazonian vertebrates deposited in the Helminthological Collection of the Oswaldo Cruz Institute with new records. Revista Brasileira de Zoologia 19:453-465.

Grundmann, A. W. 1959. Parasites recovered from six species of Utah lizards. Journal of Parasitology 45:394.

Hamilton, W. J. 1955. Notes on the ecology of the Oak toad in Florida. Herpetologica 11:205-210.

Hartdegen, R. W., and K. C. Gamble. 2002. Phillodryas baroni baroni (Baron's racer). Endoparasitism. Herpetological Review 33:141.

Harwood, P. D. 1930. A new species of Oxysomatium (Nematoda) with some remarks on the genera Oxyso- 
matium and Aplectana, and observations on the life history. Journal of Parasitology 17:61-73.

Harwood, P. D. 1932. The helminths parasitic in the Amphibia and Reptilia of Houston, Texas and vicinity. Proceedings U.S. National Museum 81:1-71.

Hill, W. C. 1941. Physaloptera terrapenis, a new nematode from a tortoise. Transactions of the American Microscopical Society 60:59-64.

Hollis, P. D. 1972. A survey of parasites of the bullfrog, Rana catesbeiana Shaw, in central East Texas. Southwestern Naturalist 17:198-200.

Ingles, L. G. 1936. Worm parasites of California Amphibia. Transactions of the American Microscopical Society 55:73-92.

Joy, J. E., and C. A. Bunten. 1997. Cosmocercoides variabilis (Nematoda: Cosmocercoidea) populations in the eastern American toad, Bufo a. americanus (Salienta: Bufonidae), from western West Virginia. Journal of the Helminthological Society of Washington 64:102-105.

Kennedy, M. J. 1977. Aplectana lynae n. sp. (Nematoda: Cosmocercidae) from the red-legged frog, Rana aurora aurora, in British Columbia. Canadian Journal of Zoology 55:630-634.

Koller, R. L., and A. J. Gaudin. 1977. An analysis of helminth infections in Bufo boreas (Amphibia: Bufonidae) and Hyla regilla (Amphibia: Hylidae) in Southern California. Southwestern Naturalist 21:503-509.

Lazell, J. D., Jr. 1964. The Lesser Antillean representatives of Bothrops and Constrictor. Bulletin of the Museum of Comparative Zoology 132:245-273.

Lehmann, D. L. 1960. Some parasites of central California amphibians. Journal of Parasitology 46:10.

Lehmann, D. L. 1965. Intestinal parasites of northwestern amphibians. Yearbook of the American Philosophical Society. pp. 284-285.

Lent, H., and J. F. T. Freitas. 1948. Uma colecao de nematodeos de vertebrados, do Museu de Historia Natural de Montevideo. Memorias do Instituto Oswaldo Cruz 46:1-71.

McAllister, C. T. 1991. Protozoan, helminth, and arthropod parasites of the spotted chorus frog, Pseudacris clarkii (Anura: Hylidae), from north-central Texas. Journal of the Helminthological Society of Washington 58:51-56.

McAllister, C. T., and C. R. Bursey. 2004. Endoparasites of the dark-sided salamander, Eurycea longicauda melanopleura, and the cave salamander, Eurycea lucifuga (Caudata: Plethodontidae), from two caves in Arkansas, U.S.A. Comparative Parasitology 71:61-66.

McAllister, C. T., and C. R. Bursey. 2005. Endoparasites of the eastern narrowmouth toad, Gastrophryne carolinensis (Anura: Microhylidae), from northwestern Louisiana and northeastern Texas, U.S.A. Comparative Parasitology 72:124-128.

McAllister, C. T., and C. R. Bursey. 2007. Some nematode and acanthocephalan parasites of the longnose leopard lizard, Gambelia wislizenii (Lacertilia: Crotaphytidae), from Arizona, California, and Texas, with a summary of helminths reported from this host. Comparative Parasitology 74:179-184.

McAllister, C. T., C. R. Bursey, and D. B. Conn. 2005. Endoparasites of Hurter's spadefoot, Scaphiopus hurterii and Plains spadefoot, Spea bombifrons (Anura:
Scaphiopoidae), from southern Oklahoma. Texas Journal of Science 57:383-389.

McAllister, C. T., C. R. Bursey, and P. S. Freed. 2010. Helminth parasites of selected amphibians and reptiles from the Republic of Ecuador. Comparative Parasitology 77:52-66.

McAllister, C. T., S. E. Trauth, and C. R. Bursey. 1995. Parasites of the pickerel frog, Rana palustris (Anura: Ranidae), from the southern part of its range. Southwestern Naturalist 40:111-116.

McAllister, C. T., S. J. Upton, and D. B. Conn. 1989. A comparative study of endoparasites in three species of sympatric Bufo (Anura: Bufonidae), from Texas. Proceedings Helminthological Society of Washington 56:162-167.

McAlpine, D. F, and M. D. B. Burt. 1998. Helminths of bullfrogs, Rana catesbeiana, green frogs, $R$. clamitans, and leopard frogs, $R$. pipiens in New Brunswick. Canadian Field-Naturalist 112:50-68.

McKenzie, V. J. 2007. Human land use and patterns of parasitism in tropical amphibian hosts. Biological Conservation 137:102-116.

Menezes, V. A., D. Vrcibradic, J. J. Vicente, G. F. Dutra, and C. R. D. Rocha. 2004. Helminths infecting the parthenogenetic whiptail lizard Cnemidophorus nativo in a restinga habitat of Bahia State, Brazil. Journal of Helminthology 78:323-328.

Molin, R. 1860. Una monograflia del genre Physaloptera. Sitxungsberichte Akademii Wissenschafter. Vienna 39: 637-672.

Molin, R. 1861. Il sottordine degli acrofalli ordiato scientificamente secondo I risultamenti delle indagini anatomiche ed embriogeniche Memorias Reale Institui Veneto Science Lettere ed Arti 9:427-633.

Moravec, F., A. Coy Otero, and V. Barus. 1990. Two remarkable nematodes from Leiocephalus sp. (Sauria: Iguanidae) from Bahamas. Helminthologia 27:225232.

Moravec, F., and H. Kaiser. 1995. Helminth parasites from West Indian frogs, with descriptions of two new species. Caribbean Journal of Science 31:252-268.

Morgan, B. B. 1943. The Physaloptera (Nematoda) of reptiles. Le Naturaliste Canadien 70:179-185.

Muzzall, P. M. 1991. Helminth infracommunities of the frogs Rana catesbeiana and Rana clamitans from Turkey Marsh, Michigan. Journal of Parasitology 77:366-371.

Muzzall, P. M., and C. R. Peebles. 1991. Helminths of the wood frog, Rana sylvatica, and spring peeper, Pseudacris c. crucifer, from southern Michigan. Journal of the Helminthological Society of Washington 58:263-265.

Odlaug, T. O. 1954. Parasites of some Ohio Amphibia. The Ohio Journal of Science 54:126-128.

O'Grady, S. P., and M. D. Dearing. 2006. Isotopic insight into host-endosymbiont relationships in liolaemid lizards. Oecologia: 150:355-361.

Ogren, R. E. 1953. A contribution to the life cycle of Cosmocercoides in snails (Nematoda: Cosmocercidae). Transactions of the American Microscopical Society 72:87-91.

Ogren, R. E. 1959. The nematode Cosmocercoides dukae as a parasite of the slug. Proceedings of the Pennsylvania Academy of Science 33:236-241. 
Ortlepp, R. J. 1922. The nematode genus Physaloptera Rud. Proceedings Zoological Society of London 72: 999-1107.

Paredes-Calderón, L., V. León-Règagnon, and L. García-Prieto. 2004. Helminth infracommunities of Rana vaillanti Brocchi (Anura: Ranidae) in Los Tuxtlas, Veracruz, Mexico. Journal of Parasitology 90:692-696.

Pearce, R. C., and W. W. Tanner. 1973. Helminths of Sceloporus lizards in the great basin and upper Colorado Plateau of Utah. Great Basin Naturalist 33:1-18.

Poinar, G. O., and O. Vaucher. 1972. Cycle larvaire de Physlaoptera retusa Rudolphi, 1819 (Nmetoda, Physalopteridae), parasite d'un lezard sud-americain. Bulleltin du Museum National D'Histoire Naturelle. 3th serie, 95:1321-1327.

Rankin, J. S. 1945. An ecological study of the helminth parasites of amphibians and reptiles of western Massachusetts and vicinity. Journal of Parasitology 31:142-150.

Rau, M. E., J. Doyle, and D. Gordon. 1978. Les parasites des animaux sauvages du Quebec. 2. Les parasites des grenouilles et des serpents de la region del l'Ile Perrot. Le Naturaliste Canadien 105:56-57.

Rau, M. E., and D. M. Gordon. 1980. Host specificity among the helminth parasites of four species of snakes. Canadian Journal of Zoology 58:929-930.

Rausch, R. 1947. Observations on some helminths parasitic in Ohio turtles. American Midland Naturalist 38:434442.

Ribas, S. C., C. R. D. Roca, P. F. Tiexeira-Filho, and J. J. Vicente. 1995. Helminths (Nematoda) of the lizard Cnemidophorus ocellifer (Sauria: Teiidae): assessing the effect of rainfall, body size and sex in the nematode infection rates. Ciencia e Cultura 47:88-91.

Ribas, S. C., C. F. D. Roca, P. F. Tiexeira-Filho, and J. J. Vicente. 1998a. Nematode infection in two sympatric lizards (Tropidurus torquatus and Ameiva ameiva) with different foraging tactics. Amphibia-Reptilia 19:323330.

Ribas, S. C., P. F. Teixeira-Filho, C. F. D. Rocha, and J. J. Vicente. 1998b. Parasitismo por nematoideos em duas especies simpatricas de Mabuya (Lacertilia: Scincidae) na restinga da Barra de Marica, R. J. Anais do VIII Seminariio Regional de Ecologia 8:883-894.

Roca, V. 1997. Tropidurus melanopleurus (NCN) Parasites. Herpetological Review 28:204.

Rocha, C. F. D. 1995. Nematode parasites of the Brazilian sand lizard, Liolaemus lutzae. Amphibia-Reptilia 16: 412-415.

Rocha, C. F. D., D. Vrcibradic, J. J. Vicente, and M. CunhaBarros. 2003. Helminths infecting Mabuya dorsivittata (Lacertilia, Scincidae) from a high-altitude habitat in Itatiaia National Park, Rio de Janeiro State, southeastern Brazil. Brazil Journal of Biology 63:129-132.

Rodriguez-Ortiz, B., L. Garcia-Prieto, and G. PerezPonce de Leon. 2003. Checklist of the helminth parasites of vertebrates in Costa Rica. Revista de Biologia Tropical 51:1-41.

Rudolphi, C. A. 1819. Entozoorum synopsis cui accedunt mantissa duplex et indices locupletissimi. Augusti Rücker, Berlin, Germany. 811 pp.

Ruiz, J. M. 1947. Revisao do genero Cruzia (Nematoda: Oxiuroidea) e estudo das especies Brasileiras. Thesis, Univesidade de Sao Paulo. 105 pp.
Savage, J. M. 2002. The Amphibians and Reptiles of Costa Rica: A Herpetofauna Between Two Continents, Between Two Seas. University of Chicago Press, Chicago, Illinois. 934 pp.

Schad, G. A. 1962. Studies on the genus Kalicephalus (Nematoda: Diaphanocephalidae). II. A taxonomic revision of the genus Kalicephalus Molin, 1861. Canadian Journal Zoology 40:1035-1165.

Schad, G. A. 1964. Studies on the genus Kalicephalus (Nematoda: Diaphanocephalidae). New records of taxonomic and ecological interest. Canadian Journal of Zoology. 42:1143-1145.

Schneider, A. 1866. Monographie der Nematoden. Reimer, Berlin. 357 pp.

Schuurmans-Stekhoven, J. H. 1950. Nematodos parasitarios del chaco Paraguayo y de Argentina del Museo de Estocolmo. Acta Zoologica Lilloana 9:325-345.

Skrjabin, K. I. 1916. [Contributions to the study of the helminthological fauna of Paraguay. I. Nematodes] Zoologicheskii Viestnik 1:705-757. (In Russian.)

Specian, R. D., and F. H. Whittaker. 1980. A survey of the helminth fauna of Cnemidophorus murinus from the Island of Curacao. Proceedings of the Helminthological Society of Washington 47:275-276.

Sprehn, C. E. W. 1929. Eine neue Ascaride, Ophidascaris arndi n. sp. aus einer südamerikanischen Schlange. Zoologischer Anzeiger 83:280-282.

Sprehn, C. 1932. Uber einige von Dr. Eisentraut in Bolivien gesammelte nematoden. Zoologischer Anzeiger. 100: 273-284.

Sprent, J. F. A. 1978. Ascaridoid nematodes of amphibians and reptiles: Polydelphis, Travassosascaris n. g. and Hexametra. Journal of Helminthology 52:355-384.

Sprent, J. F. A. 1979. Ascaridoid nematodes of amphibians and reptiles: Terranova. Journal of Helminthology 53: 265-282.

Sprent, J. F. A. 1988. Ascaridoid nematodes of amphibians and reptiles: Ophidascaris Baylis, 1920. Systematic Parasitology 11:165-213.

Telford, S. R. 1965. Some observations on the effects of varying ambient temperatures in vivo on filarial worms of snakes. Japanese Journal of Experimental Medicine 35:291-300.

Telford, S. R. 1970. A comparative study of endoparasitism among some southern California lizard populations. American Midland Naturalist 83:516-554.

Tinsley, R. C. 1990. The influence of parasite infection on mating success in spadefoot toads, Scaphiopus couchii. American Zoologist 30:313-324.

Travassos, L. 1931. Pesquizas helminthologicas realizadas em Hamburgo. IX. Ensaio monographico da familia Cosmocercidae Trav., 1925 (Nematoda). Memórias do Instituto Oswaldo Cruz 25:235-298.

Trowbridge, A. H., and H. M. Hefley. 1934. Preliminary studies of the parasite fauna of Oklahoma anurans. Proceedings of the Oklahoma Academy of Science 14: 16-19.

Ubelaker, J. E., and M. D. Dailey. 1966. Observations on Kalicephallus subulatus Molin, 1861 from Nicaragua and Mexico. Revista de Biologia tropical 14:47-53.

Vanderburgh, D. J., and R. C. Anderson. 1987a. Preliminary observations on seasonal changes in prevalence and intensity of Cosmocercoides variabilis (Nematoda: Cosmocercoidea) in Bufo americanus 
(Amphibia). Canadian Journal of Zoology 65:16661667.

Vanderburgh, D. J., and R. C. Anderson. 1987b. The relationship between nematodes of the genus Cosmocercoides Wilkie, 1930 (Nematoda: Cosmocercoidea) in toads (Bufo americanus) and slugs (Deroceras laeve). Canadian Journal Zoology 65:1650-1661.

Vaz, Z. 1938. Nova especie do genero Ophidascaris parasita da cascavel (Crotalus terrificus). Livro jubilar do Professor Lauro Travassos: editado para coommenmorar o $25^{\circ}$ anniversario de suas actividades scientificas (1913-1938). Typographia do Instituto Osalado Cruz, Rio de Janeiro, Brazil. pp. 495-499.

Vicente, J. J. 1981. Helmintos de Tropidurus (Lacertilia, Iguanidae) da colecao helmintologica do Intituto Oswaldo Cruz. II. Nematoda. Atas Sociedade de Biologia de Rio de Janeiro 22:7-18.

Vicente, J. J., and C. R. Jardim. 1980. Filarideos da Colecao helmintologica do Instituto Oswaldo Cruz. I. Peixes, anfibios e repteis. Atas Sociedad Biologia. Rio de Janeiro 21:47-57.

Vicente, J. J., H. O. Rodrigues, D. C. Gomes, and R. M. Pinto. 1993. Nematóides do Brazil. Parte III: Nematóides de répteis. Revista brasileria de Zoologia 10:19-168.

Vicente, J. J., and E. Santos. 1967. Ocorrencia de Physaloptera retusa Rudolphi, 1819 em novo hospedeiro (Nematoda, Physalopteridae). Atas da Sociedade de Biologia do Rio de Janeiro 11:75-77.

Viquez, S. C. 1935. Animales venenosos de Costa Rica. Parásitos intestinales de nuestros animals encontrados en sus inestigaciones por el Licenciado Carlos Viquez. Imprenta Nacional, San José, Costa Rica. 313 pp.

Vrcibradic, D., M. Cunha-Barros, J. J. Vicente, C. A. C. Galdino, F. H. Hatano, M. Van Sluys, and C. F. D.
Rocha. 2000. Nematode infection patterns in four sympatric lizards from a restinga habitat (Jurubatiba) in Rio de Janeiro state, southeastern Brazil. AmphibiaReptilia 21:307-316.

Vrcibradic, D., C. F. D. Rocha, C. R. Bursey, and J. J. Vicente. 2002. Helminth communities of two sympatric skinks (Mabuya agilis and Mabuya macrorhyncha) from two 'restinga' habitats in southeastern Brazil. Journal of Helminthology 76:355-361.

Vrcibradic, D., C. F. D. Rocha, M. van Sluys, and C. R. Bursey. 2001 Mabuya macrorhyncha (NCN) Endoparasites. Herpetological review 32:256-257.

Waitz, J. A. 1961. Parasites of Idaho amphibians. Journal of Parasitology 47:89.

Walker, K. A., and D. V. Matthias. 1973. Helminths of some northern Arizona lizards. Proceedings of the Helminthological Society of Washington 40:168-169.

Walton, A. C. 1929. Studies on some nematodes of North American frogs. Journal of Parasitology 15:227-240.

Walton, A. C. 1938. The Nematoda as parasites of Amphibia. IV. Transactions of the American Microscopical Society 57:38-53.

Walton, A. C. 1947. Parasites of the Ranidae (Amphibia) I. Journal of Parasitology 33:25-26.

Wilkie, J. S. 1930. Some parasitic nematodes from Japanese Amphibia. Annals and Magazine of Natural History, Series 10, 6:606-614.

Woodbury, L. A. 1934. Notes on some parasites of three Utah reptiles. Copeia 1934:51-52.

Yoder, H. R., and J. R. Coggins. 2007. Helminth communities in five species of sympatric amphibians from three adjacent ephemeral ponds in southeastern Wisconsin. Journal of Parasitology 93:755-760. 